\title{
$N$-representability problem within the framework of the contracted Schrödinger equation
}

\author{
C. Valdemoro, ${ }^{1}$ L. M. Tel, ${ }^{2}$ and E. Pérez-Romero ${ }^{2}$ \\ ${ }^{1}$ Instituto de Matemáticas y Física Fundamental, CSIC, Serrano 123, 28006-Madrid, Spain \\ ${ }^{2}$ Departamento de Química-Física, Universidad de Salamanca, 37008-Salamanca, Spain \\ (Received 16 March 1998; revised manuscript received 28 June 1999; published 17 February 2000)
}

\begin{abstract}
A a procedure is proposed by which, in the course of an iterative solution of the second-order contracted Schrödinger equation, The $N$ representability of the second-order reduced density matrix can be tested with increasing stringency. This procedure was suggested by an extended study of the $G$ conditions and from the contraction into the two-electron space of an $N$-fermion relation, expressing in matrix form the antisymmetry and normalization properties of the $N$-electron wave function. Several relations are reported.
\end{abstract}

PACS number(s): $31 \cdot 10 .+\mathrm{z}$

\section{INTRODUCTION}

To determine a reduced density matrix $(\mathrm{RDM})[1-4]$ directly without a previous knowledge of the corresponding Wave Function became one of the objectives in molecular physics and quantum chemistry during the 1960s. The difficulty, as Coleman pointed out in his basic paper [5] in 1963, was that since these matrices originate from an $N$-electron wave function they must have well-defined properties. The search for the constraints to be imposed when varying the expression of the energy as a trace of the product of the second order reduced Hamiltonian and a 2-RDM defined, in practice, the $N$-representability problem. Thus, if this matrix was an $N$-representable 2-RDM one should obtain an upper bound for the ground-state energy. The solution of the problem thus defined became the aim of many studies [6-27] which were mainly centered on investigating the topology and the group-theoretical properties of RDM's. Both Coleman [28] and Löwdin [29] published comprehensive reviews of the rich bibliography generated in this field up to 1985 .

The possibility of solving iteratively the second order contracted Schrödinger equation (CSE) [30-39], which has been investigated in the last years, suggests a less restrictive and more general way of looking at this problem since the variational conditions are already taken into account by the CSE itself [40-42].

After a section devoted to formal definitions, this new situation is analyzed, and the problem redefined in Sec. III. The problem then becomes a search for a reliable test which a matrix - represented in a two-electron space, and obtained through the iterative solution of the 2-CSE (it-CSE) - must satify so that it may be considered $N$-representable, or closely so.

The rest of the paper is dedicated to this investigation. Its structure is based on a formal partitioning of the 2-RDM's necessary properties into two classes.

(i) Those properties directly following from the structure of the density operator. To this category correspond the so called $G$ conditions which are studied in Sec. IV. In this section some well-known $G$ conditions are rewritten in terms of the virtual excitations of the system. It is shown that the second order $G$ matrix (and, by extension, the higher-order ones) determines the electron correlation contribution to the 2-RDM (and higher-order RDM's). The study of the second-, third-, and fourth-order $G$ matrices, of their properties and of their interrelation with the RDM's, leads to a set of new relations which establish bounds to each one of the elements of an $N$-representable 2-RDM. Finally, the fact that the second-order $G$ matrix must be positive implies conditions on the average of the 2-RDM elements, diagonal and off-diagonal.

(ii) The matrix considered must be derived through an integration process from an antisymmetric $\mathrm{N}$-electron function. The conditions that this implies are found by considering the following.

(a) The properties of a well-behaved antisymmetric $\mathrm{N}$-electron wave function can be represented in the $N$-electron space in terms of particles and holes RDM's by an $N$-fermion relation ( $N$-FR). This is shown in Sec. V.

(b) The global integration or matrix contraction of the $N$-FR — carried out in Sec. VI-into the $p$ space generates a family of fermion relations ( $p$-FR) which by construction possess a primitive (or pre image): the $N$-FR

In Sec. VII, we argue that the set of conditions established in the study of these two interrelated classes of properties constitute a reliable procedure for testing the $N$-representability of a 2-RDM obtained through the it-CSE. This testing procedure includes a set of conditions linking the 2-RDM with the 3- and 4-RDM's.

In order to perform the matrix contraction of the $N$-FR we developed a series of mathematical tools which may also prove useful in a more general context for operating with RDM's. These technical developments are needed so as to render a self-contained paper; but they are gathered in the Appendixes to make reading the paper easier.

\section{NOTATION}

All through this work we will assume that the number of orthonormal spin orbitals of our basis set, $2 K$, has a large but finite value. Thus we limit ourselves here to problems that can be modeled in such a finite space. In addition, in order to simplify the developments, we also assume that all our matrices are real.

We will also consider here that the system under study has a fixed number of electrons $N$. The space of all the states of our system can therefore be spanned by a basis of 
$N$-electron normalized Slater determinants built up from the spin-orbital basis.

In all the developments $\mathcal{L}$ represents any given antisymmetrized, normalized $N$-electron state. It can be any state, pure or ensemble, provided that it can be expressed as a normalized linear combination of the Slater determinants defined above. In addition, in some sections $\mathcal{L}$ will be required to satisfy the Schrödinger equation and, hence, to be an eigenstate of the Hamiltonian.

In the occupation number representation, which is the representation which will be used here, the $q$-order reduced density matrix corresponding to a state $\mathcal{L}$ is

$$
{ }^{q} \mathrm{D}_{i_{1} i_{2} \ldots i_{q} ; j_{1} j_{2} \ldots j_{q}}^{\mathcal{L}}=\frac{1}{q !}\left\langle\mathcal{L}\left|\mathrm{b}_{i_{1}}^{\dagger} \mathrm{b}_{i_{2}}^{\dagger} \ldots \mathrm{b}_{i_{q}}^{\dagger} \mathrm{b}_{j_{q}} \ldots \mathrm{b}_{j_{2}} \mathrm{~b}_{j_{1}}\right| \mathcal{L}\right\rangle
$$

where the $\mathrm{b}_{r}^{\dagger} / \mathrm{b}_{r}$ operators create and annihilate an electron in the spin orbital $r$.

Similarly, the $q$-order holes reduced density matrix (9HRDM) is

$$
{ }^{q} \overline{\mathrm{D}}_{i_{1} i_{2} \ldots i_{q} ; j_{1} j_{2} \ldots j_{q}}^{\mathcal{L}}=\frac{1}{q !}\left\langle\mathcal{L}\left|\mathrm{b}_{j_{q}} \ldots \mathrm{b}_{j_{2}} \mathrm{~b}_{j_{1}} \mathrm{~b}_{i_{1}}^{\dagger} \mathrm{b}_{i_{2}}^{\dagger} \ldots \mathrm{b}_{i_{q}}^{\dagger}\right| \mathcal{L}\right\rangle .
$$

Note that the holes are not relative to any independent particle state (as in other many-body approaches), but they are relative to the state $\mathcal{L}$.

When the bra and ket in Eq. (1) [or Eq. (2)] do not refer to the same state we have a transition RDM. In such a case, both the bra and ket identifications appear in the text as super indices. Superscripts referring to states will be suppressed if no ambiguity arises.

When sequences of several creator and annihilator operators appear in a formula, as in Eqs. (1) or (2), it is very convenient to handle them in a global way. Hence, we define the global operators as

$$
{ }^{q} \mathrm{~B}_{\Lambda}^{\dagger} \equiv \mathrm{b}_{i_{1}}^{\dagger} \mathrm{b}_{i_{2}}^{\dagger} \ldots \mathrm{b}_{i_{q}}^{\dagger}, \quad{ }^{q} \mathrm{~B}_{\Omega} \equiv \mathrm{b}_{j_{q}} \ldots \mathrm{b}_{j_{2}} \mathrm{~b}_{j_{1}},
$$

where $i_{1}<i_{2}<\cdots<i_{q}$ and $j_{1}<j_{2}<\cdots<j_{q}$. That is,

$$
{ }^{q} \mathrm{~B}_{\Lambda}^{\dagger}|0\rangle=|\Lambda\rangle \equiv\left|i_{1} i_{2} \ldots i_{q}\right\rangle .
$$

The algebra of these operators is reported in Appendix B.

With this notation, the $q$-RDM and the $q$-HRDM are

$$
\begin{aligned}
{ }^{q} \mathrm{D}_{\Lambda ; \Omega}^{\mathcal{L}} & =\left\langle\mathcal{L}\left|{ }^{q} \mathrm{~B}_{\Lambda}^{\dagger}{ }^{q} \mathrm{~B}_{\Omega}\right| \mathcal{L}\right\rangle, \\
{ }^{q} \overline{\mathrm{D}}_{\Lambda ; \Omega}^{\mathcal{L}} & =\left\langle\mathcal{L}\left|{ }^{q} \mathrm{~B}_{\Omega}{ }^{q} \mathrm{~B}_{\Lambda}^{\dagger}\right| \mathcal{L}\right\rangle,
\end{aligned}
$$

respectively.

Note that the ordering of the indices eliminates the denominator $q$ ! and that, when more than two subscripts are needed to denote a matrix element, a semicolon separates the row labels from the column labels. Another general feature of the RDM and HRDM notation is that the creator subscripts define the rows [see the left-hand side of Eqs. (1) and (2)].

\section{III. $N$-REPRESENTABILITY PROBLEM WITHIN THE FRAMEWORK OF THE CONTRACTED SCHRÖDINGER EQUATIONS: TWO ENERGY EXPRESSIONS}

A $p$-CSE $[40,41]$ is the integration of the Schrödinger equation over $N-p$ electron variables or, equivalently, the result of contracting the matrix representation of the Schrödinger equation from the $N$ electron space into the $p$-electron one [42]. The $p$-CSE must be simultaneously satisfied by the energy $E$ and the $p-,(p+1)$-, and $(p+2)$-RDM's corresponding to a given eigenstate $\mathcal{L}$ of the $N$-electron system.

In this notation, a diagonal element of the second order CSE takes the form

$$
\begin{aligned}
E_{\mathcal{L}}{ }^{2} \mathrm{D}_{p q ; p q}^{\mathcal{L}}= & \left({ }^{0} \mathbf{H}^{2} \mathbf{D}^{\mathcal{L}}\right)_{p q ; p q}+3 \sum_{i, l, k}\left({ }^{0} \mathrm{H}_{i q ; k l}{ }^{3} \mathrm{D}_{p q i ; p l k}^{\mathcal{L}}\right. \\
& \left.+{ }^{0} \mathrm{H}_{i p ; k l}{ }^{3} \mathrm{D}_{p q i ; l q k}^{\mathcal{L}}\right)+6 \sum_{i, j, k, l}{ }^{0} \mathrm{H}_{i j ; k l}{ }^{4} \mathrm{D}_{p q i j ; p q k l}^{\mathcal{L}},
\end{aligned}
$$

where ${ }^{0} \mathrm{H}$ is [43]

$$
{ }^{0} \mathrm{H}_{i j ; k l}=\{i k \mid j l\}+\frac{\left(\delta_{i k} \epsilon_{j l}+\delta_{j l} \epsilon_{i k}\right)}{N-1},
$$

and where the symbol $\epsilon$ represents the one-electron integrals and $\{i k \mid j l\}$ is a two-electron integral in Mulliken's notation. Equation (6) is a member of a family of relations linking elements of RDM's of various orders. The CSE relates them through the Hamiltonian. Other such families of relations will be dealt with later on.

Equation (6) seems to indicate that the energy $E_{\mathcal{L}}$ is a function of the 2-, 3-, and 4-RDM's. On the other hand, it is well known that

$$
E_{\mathcal{L}}=\operatorname{tr}\left({ }^{0} \mathbf{H}^{2} \mathbf{D}^{\mathcal{L}}\right)
$$

This result can also be recovered from Eq. (6) by summing over the $p$ and $q$ indices. Thus

$$
\begin{aligned}
E_{\mathcal{L}}\left(\begin{array}{l}
N \\
2
\end{array}\right) & =\operatorname{tr}\left({ }^{0} \mathbf{H}^{2} \mathbf{D}^{\mathcal{L}}\right)\left(1+2(N-2)+\frac{(N-3)(N-2)}{2}\right) \\
& \equiv \operatorname{tr}\left({ }^{0} \mathrm{H}^{2} \mathbf{D}^{\mathcal{L}}\right)\left(\begin{array}{l}
N \\
2
\end{array}\right) .
\end{aligned}
$$

When the 2-RDM corresponding to an eigenstate is unknown, the structure of both Eqs. (6) and (9) tell us that the 3- and 4-RDM's must be explicitly taken into account. Therefore, although the information contained in a 2-RDM determines the expectation value of the energy of the corresponding state (any state), the information carried not only by the 2-RDM but also by the 3- and 4-RDM's is needed in order to determine the energy and the 2-RDM corresponding to an eigenstate. In other words, Eq. (6) expresses the variational conditions that the energy and the 2-, 3-, and 4-RDM's must fulfill. 
In order to solve Eq. (6), an iterative procedure has been proposed (it-CSE) [30,31]. The main features of our approach are the following.

(i) The 3-RDM and 4-RDM are approximated in terms of two initial trial RDM's of first and second orders, respectively.

(ii) The trials for the 2-, 3-, and 4-RDM's thus obtained are replaced on the right-hand side of Eq. (6) which becomes a matrix represented in the two-electron space, $\mathcal{M}_{0}$, such that

$$
E^{2} \mathrm{D}=\mathcal{M}_{0} .
$$

(iii) Then

$$
\operatorname{tr}\left(\mathcal{M}_{0}\right)=E\left(\begin{array}{l}
N \\
2
\end{array}\right)
$$

hence

$$
E_{0}=\frac{\operatorname{tr}\left(\mathcal{M}_{0}\right)}{\left(\begin{array}{l}
N \\
2
\end{array}\right)}, \quad{ }^{2} \mathrm{D}_{0}=\frac{\operatorname{tr}\left(\mathcal{M}_{0}\right)}{E_{0}} .
$$

(iv) After contracting ${ }^{2} \mathrm{D}_{0}$ to obtain the corresponding ${ }^{1} \mathrm{D}_{0}$, the second iteration starts by calculating new trials for the 3- and 4-RDM.

(v) The procedure is repeated until convergence is obtained.

Evidently, the crucial question is how accurately the 3and 4-RDM's may be constructed from the knowledge of the 1- and 2-RDM's. Several approaches have been described for solving this problem. Thus, Valdemoro and co-workers based their development on the particle-hole equivalence, the $N$-representability conditions, and the spin-symmetry conditions [31,33]; Nakatsuji and co-workers $[32,34,44]$ added a term to the zero-order algorithm of Valdemoro and coworkers by applying a perturbative expansion for high-order RDM's similar to the Green function one. Mazziotti proposed to approximate the high-order RDM's by applying cumulant theory $[35,36]$ and obtained the same formal expression as Nakatsuji and co-workers for the 4-RDM. It is in the calculation of the third-order term of these expansions, as well as in the estimation of the remaining error, where the differences among the three approaches are more significant. Both Valdemoro and co-workers and Mazziotti emphasized the need of consistency through contraction of the 4-, 3- and 2-RDM's entering the 2-CSE. An important feature of the proposal of Mazziotti is that he showed the great advantage of working in a basis of natural orbitals [45]. Although further studies will no doubt improve the accuracy of the method, the results so far achieved with the three approaches are highly satisfactory [31-35].

A very interesting feature is that while the zero-order algorithm for approximating the 3-RDM, common to these three different approaches, provides very accurate values for most elements there are some other where the higher cumulant or perturbative terms must be included.

The cause of this nonhomogeneity was recently analyzed by our group [38]. The results point out that only those matrix elements whose orbitals belong to a small subset of or- bitals give significant errors when evaluated with the zeroorder approximation. The orbitals belonging to this subset can be characterized as generalized frontier orbitals by analogy with the meaning of this term when considering a Hartree-Fock molecular ground state. In these single configuration states, the highest (in energy) occupied molecular orbital and lowest (in energy) empty molecular orbital are called frontier orbitals. This concept may be extended and applied to states with a dominant configuration. Then, only those-comparatively few-RDM elements, involving the highest occupied and the lowest empty spin orbitals for every symmetry species, require a specialised treatment.

Among the questions which the it-CSE procedure raises, the most important are the following

(i) How to test the $N$-representability of the 2-RDM resulting from the iterative process.

(ii) To determine whether any additional conditions must be introduced when approximating the 3- and 4-RDM's in order to make sure that the average contributions of these higher-order matrices to the 2-RDM is $N$ representable. As will be seen in Sec. IV it is at this level that the definition of the generalized frontier orbitals may be very useful.

These are the main questions studied in the present paper. Our leading idea is that the $N$-representability problem concerning a 2-RDM may be analyzed by decomposing it into two different mathematical problems:

(1) The matrix must represent a two-density operator. (2) The matrix considered must be derived through an integration process from an antisymmetric $N$-electron function. That is, there are properties following directly from the properties of the operators in expression (1), and there are properties determined by the bra and ket of Eq. (1). We are evidently aware that this formal decomposition is artificial, since the two problems are closely interrelated; however, it permits us to assume that, when the two sets of necessary conditions resulting from this analysis are simultaneously satisfied by a given two-order matrix, it is safe to assume that this matrix is $N$ representable, or closely so.

\section{DENSITY CONDITIONS}

The hermiticity and positivity [3] of the RDM's are properties following directly from their definition, [Eq. (1)], and from the properties of the one-electron operators. The values of the traces, the so called $G$ conditions [10] and the antisymmetry are properties which also reflect the density characteristics of the operators but are not so immediate. In this section we will consider the RDM traces and the $G$ conditions in some detail. As will be seen, a study of the $G$ conditions leads to the expression of the bounds on the correlation effects of the RDM elements; it also leads to relations involving the square of the 2-RDM, which state what may be considered the image, in the reduced space, of the idempotency property of the density matrix ( $N$-RDM). The antisymmetry of the RDM's is treated in Sec. IV A.

\section{A. Traces}

A well-known basic relation of the one-electron density operator is 


$$
\sum_{i} \mathrm{~b}_{i}^{\dagger} \mathrm{b}_{i}=\hat{N}=\hat{1} N
$$

hence

$$
\hat{1}=\sum_{i} \frac{b_{i}^{\dagger} b_{i}}{N} .
$$

This defines the first-order unit operator which is the simplest of a family of unit operators operating upon $\mathrm{N}$-electron states. Thus, in general

$$
{ }^{p} \hat{1}=\sum_{\Lambda} \frac{{ }^{p} \mathrm{~B}_{\Lambda}^{\dagger p} \mathrm{~B}_{\Lambda}}{\left(\begin{array}{l}
N \\
p
\end{array}\right)} .
$$

In a similar way, one can define a family of unit operators of the hole-density type,

$$
{ }^{p} \hat{1}=\sum_{\Lambda} \frac{{ }^{p} \mathrm{~B}_{\Lambda}{ }^{p} \mathrm{~B}_{\Lambda}^{\dagger}}{\left(\begin{array}{c}
2 K-N \\
p
\end{array}\right)},
$$

where we recall that we are assuming that $K$ is a finite number.

Relations (4) and (13) imply that

$$
\operatorname{tr}^{p} \mathbf{D}=\left(\begin{array}{c}
N \\
p
\end{array}\right) .
$$

Similarly, one has

$$
\operatorname{tr}^{p} \overline{\mathbf{D}}=\left(\begin{array}{c}
2 K-N \\
p
\end{array}\right)
$$

In fact, the operations with the HRDM are similar to those involving RDM's the only difference being that the factor $2 K-N$ replaces $N$. Obviously for $K=\rightarrow \infty$ all the HRDM traces blow up.

\section{B. $G$ conditions}

Coleman and Erdahl draw our attention to the importance of explicitly imposing the positivity of the $G$ matrices when testing the $N$ representability of the RDM's. Here we will study some questions that the two basic papers on $G$ conditions by Garrod and Percus [10] and Weinhold and Wilson [17] suggested to us considering our latest results on the correlation effects $[37,38]$.

\section{Second-order $G$ condition}

The simplest second-order $G$ condition is

$$
{ }^{2} g_{i, j ; i, j}^{\mathcal{L}} \equiv\left\langle\mathcal{L}\left|\mathrm{b}_{i}^{\dagger} \mathrm{b}_{j} \mathrm{~b}_{j}^{\dagger} \mathrm{b}_{i}\right| \mathcal{L}\right\rangle={ }^{1} \mathrm{D}_{i ; i}-2 !^{2} \mathrm{D}_{i j ; i j} \geqslant 0 .
$$

Following Garrod and Percus [10], the definition of an element of a more general positive $G$ matrix is

$$
{ }^{2} G_{j, l ; k, i}^{\mathcal{L}}=\left\langle\mathcal{L}\left|\mathrm{b}_{j}^{\dagger} \mathrm{b}_{l} \mathrm{~b}_{i}^{\dagger} \mathrm{b}_{k}\right| \mathcal{L}\right\rangle-\left\langle\mathcal{L}\left|\mathrm{b}_{j}^{\dagger} \mathrm{b}_{l}\right| \mathcal{L}\right\rangle\left\langle\mathcal{L}\left|\mathrm{b}_{i}^{\dagger} \mathrm{b}_{k}\right| \mathcal{L}\right\rangle .
$$

Considering the set of $\mathcal{L}^{\prime}$ states such that

$$
|\mathcal{L}\rangle\left\langle\mathcal{L}\left|+\sum_{\mathcal{L}^{\prime} \neq \mathcal{L}}\right| \mathcal{L}^{\prime}\right\rangle\left\langle\mathcal{L}^{\prime}\right|=\hat{\mathbf{I}},
$$

the previous equation may be rewritten as

$$
\begin{aligned}
{ }^{2} G_{j, l ; k, i}^{\mathcal{L}} & \equiv \sum_{\mathcal{L}^{\prime} \neq \mathcal{L}}\left\langle\mathcal{L}\left|\mathrm{b}_{j}^{\dagger} \mathrm{b}_{l}\right| \mathcal{L}^{\prime}\right\rangle\left\langle\mathcal{L}^{\prime}\left|\mathrm{b}_{i}^{\dagger} \mathrm{b}_{k}\right| \mathcal{L}\right\rangle \\
& \equiv \sum_{\mathcal{L}^{\prime} \neq \mathcal{L}}{ }^{1} \mathrm{D}_{j ; l}^{\mathcal{L} \mathcal{L}^{\prime} 1}{ }^{1} \mathrm{D}_{i ; k}^{\mathcal{L}^{\prime} \mathcal{L}} .
\end{aligned}
$$

Note, that, as mentioned at the begining of Sec. II, the semicolon appearing in the subscripts separates the row and column labels, and when denoting elements of the $g$ and $G$ matrices we have introduced a comma to separate the indices referring to creators from those referring to annihilators.

The elements of this Hermitian $G$ matrix are those of a matrix describing pure two-body effects through the products of transition RDM's elements corresponding to all the virtual excitations. There is an interesting and not too obvious feature attached to this matrix. Let us now recall the expression for the 2-RDM [37]:

$$
2 !{ }^{2} \mathrm{D}_{j i ; l k}={ }^{1} \mathrm{D}_{j ; l}{ }^{1} \mathrm{D}_{i ; k}-\delta_{i, l}{ }^{1} \mathrm{D}_{j ; k}+\sum_{\mathcal{L}^{\prime} \neq \mathcal{L}}{ }^{1} \mathrm{D}_{j ; l}^{\mathcal{L} \mathcal{L}^{\prime}{ }^{1} \mathrm{D}_{i ; k}^{\mathcal{L}} \mathcal{L}^{\mathcal{L}}}
$$

$$
={ }^{1} \mathrm{D}_{j ; l}{ }^{1} \mathrm{D}_{i ; k}-{ }^{1} \mathrm{D}_{i ; l}{ }^{1} \mathrm{D}_{j ; k}-{ }^{1} \overline{\mathrm{D}}_{i ; l}{ }^{1} \mathrm{D}_{j ; k}+\sum_{\mathcal{L}^{\prime} \neq \mathcal{L}}{ }^{1} \mathrm{D}_{j ; l}^{\mathcal{L} \mathcal{L}^{\prime}{ }^{1} \mathrm{D}_{i ; k}^{\mathcal{L}}{ }^{\mathcal{L}}} .
$$

The last two terms describe the correlation effects in a 2-RDM element, particularly the last term, the one describing the pure two-body effects defining what we will refer to here as the "correlation matrix." Hence, Eq. (21) may be expressed as a function of the $2-G$ matrix:

$$
2 !{ }^{2} \mathrm{D}_{j i, l k}={ }^{1} \mathrm{D}_{j ; l}{ }^{1} \mathrm{D}_{i ; k}-\delta_{i, l}{ }^{1} \mathrm{D}_{j ; k}+{ }^{2} G_{j, l ; k, i} .
$$

It should be stressed that, while $G>0$, no such property applies in principle to the correlation matrix. It must be underlined that $G$ and the correlation matrix are built up by the same elements, but the ordering of the elements is different.

Obviously, if one is testing the $N$ representability of a given second-order matrix, the corresponding $G$ matrix can immediately be deduced from Eq. (22) and subsequently diagonalized in order to check its positivity. Since the ensemble $N$-representability conditions for the 1-RDM are known, this would be a global test for the correlation terms of the 2-RDM element. We refer to this test as global because it simultaneously concerns to all the 2-RDM elements. As will be seen below, there are a number of relations involving higher-order matrices which condition the values of each 2-RDM element. 


\section{Bounds for the 2-RDM diagonal elements}

In order to analyze more closely the conditions that the 2-RDM diagonal elements should satisfy, let us consider the conditions derived from the positivity of the $G$-diagonal elements. Since

$$
\begin{aligned}
{ }^{2} G_{i, j ; i, j}^{\mathcal{L}} & =\left\langle\mathcal{L}\left|\mathrm{b}_{i}^{\dagger} \mathrm{b}_{j} \mathrm{~b}_{j}^{\dagger} \mathrm{b}_{i}\right| \mathcal{L}\right\rangle-\left\langle\mathcal{L}\left|\mathrm{b}_{i}^{\dagger} \mathrm{b}_{j}\right| \mathcal{L}\right\rangle\left\langle\mathcal{L}\left|\mathrm{b}_{j}^{\dagger} \mathrm{b}_{i}\right| \mathcal{L}\right\rangle \\
& \equiv{ }^{2} g_{i, j ; i, j}^{\mathcal{L}}-\left({ }^{1} \mathrm{D}_{i ; j}\right)^{2} \geqslant 0,
\end{aligned}
$$

one has

$$
{ }^{1} \mathrm{D}_{i ; i}-2 !{ }^{2} \mathrm{D}_{i j ; i j}-\left({ }^{1} \mathrm{D}_{i ; j}\right)^{2} \geqslant 0 \text {. }
$$

This second condition is more exacting than the simple $g$ condition given by Eq. (17), because $\left({ }^{1} \mathrm{D}_{i ; j}\right)^{2}$ is either positive or null.

Now, considering Eq. (24) and recalling the expressions for the 2-RDM [Eq. (21)] and 2-HRDM as functions of the two-body correlation matrices [37]

$$
\begin{aligned}
& 2 !{ }^{2} \mathrm{D}_{i j ; i j}={ }^{1} \mathrm{D}_{i ; i}{ }^{1} \mathrm{D}_{j ; j}+\sum_{\mathcal{L}^{\prime} \neq \mathcal{L}}{ }^{1} \mathrm{D}_{i ; i}^{\mathcal{L}{ }^{\prime}{ }^{1}} \mathrm{D}_{j ; j}^{\mathcal{L}^{\prime} \mathcal{L}} \geqslant 0, \\
& 2 !{ }^{2} \overline{\mathrm{D}}_{i j ; i j}={ }^{1} \overline{\mathrm{D}}_{i ; i}{ }^{1} \overline{\mathrm{D}}_{j ; j}+\sum_{\mathcal{L}^{\prime} \neq \mathcal{L}}{ }^{1} \mathrm{D}_{i ; i}^{\mathcal{L} \mathcal{L}^{\prime}{ }^{1} \mathrm{D}_{j ; j}^{\mathcal{L}}{ }^{\prime} \mathcal{L} \geqslant 0,}
\end{aligned}
$$

and expressing the factor 1 multiplying ${ }^{1} \mathrm{D}_{i ; i}$ in Eq. (24) as ${ }^{1} \mathrm{D}_{j ; j}+{ }^{1} \overline{\mathrm{D}}_{j ; j}$, we may now rewrite relation (24) and express it as an upper bound for the virtual excitations contribution to the diagonal elements of the 2-RDM-the correlation matrix

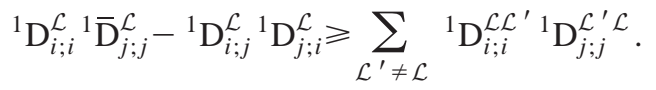

Moreover, two lower bounds for this same term follow from Eqs. (25) and (26):

$$
\begin{aligned}
& -{ }^{1} \mathrm{D}_{i ; i}^{\mathcal{L}}{ }^{1} \mathrm{D}_{j ; j}^{\mathcal{L}} \leqslant \sum_{\mathcal{L}^{\prime} \neq \mathcal{L}}{ }^{1} \mathrm{D}_{i ; i}^{\mathcal{L}{ }^{\prime}{ }^{\prime}} \mathrm{D}_{j ; j}^{\mathcal{L}{ }^{\prime} \mathcal{L}}, \\
& -{ }^{1} \overline{\mathrm{D}}_{i ; i}^{\mathcal{L}}{ }^{1} \overline{\mathrm{D}}_{j ; j}^{\mathcal{L}} \leqslant \sum_{\mathcal{L}^{\prime} \neq \mathcal{L}}{ }^{1} \mathrm{D}_{i ; i}^{\mathcal{L} \mathcal{L}^{\prime}{ }^{1} \mathrm{D}_{j ; j}^{\mathcal{L}}{ }^{\prime} \mathcal{L}} .
\end{aligned}
$$

In general, when the absolute value of the left-hand side of Eq. (28) is large, the absolute value of Eq. (29) is small and vice versa. Therefore, Eqs. (27), (28), and (29) generally impose very precise bounds on the term accounting for the correlation effects in the 2-RDM diagonal elements. Note, however, that the bounds may be less limiting for the elements whose indices correspond to frontier orbitals because, in this case, the negative quantities appearing in the left-hand side of Eqs. (28) and (29) will not have negligible absolute values.

\section{2-RDM off-diagonal elements with three different indices}

By extending the previous arguments, let us therefore consider the third-order $G$ matrix which should also be positive, and whose generic element is defined as

$$
\begin{aligned}
{ }^{3} G_{i, j, k ; q, p, l}= & \sum_{\mathcal{L}^{\prime}, \mathcal{L}^{\prime \prime} \neq \mathcal{L}}\left\langle\mathcal{L}\left|\mathrm{b}_{i}^{\dagger} \mathrm{b}_{j}\right| \mathcal{L}^{\prime}\right\rangle\left\langle\mathcal{L}{ }^{\prime}\left|\mathrm{b}_{k}^{\dagger} \mathrm{b}_{l}\right| \mathcal{L}^{\prime \prime}\right\rangle \\
& \times\left\langle\mathcal{L}^{\prime \prime}\left|\mathrm{b}_{p}^{\dagger} \mathrm{b}_{q}\right| \mathcal{L}\right\rangle .
\end{aligned}
$$

It has been interpreted [37] that these elements are pure three-body correlation terms, Also appearing with a different ordering of the indices, as part of the 3-RDM. Let us consider the diagonal case and replace $\left|\mathcal{L}^{\prime}\right\rangle\left\langle\mathcal{L}^{\prime}\right|$ by $\hat{\mathbf{1}}-|\mathcal{L}\rangle\langle\mathcal{L}|$ and similarly for $\left|\mathcal{L}^{\prime \prime}\right\rangle\left\langle\mathcal{L}^{\prime \prime}\right|$. Then, using the same kind of algebraic manipulations as above, the following inequality is obtained:

$$
\left({ }^{1} \mathrm{D}_{i ; j}\right)^{2}{ }^{1} \mathrm{D}_{k ; k}+2 !{ }^{2} \mathrm{D}_{i k ; i k}-22 !{ }^{2} \mathrm{D}_{i k ; j k}{ }^{1} \mathrm{D}_{i ; j} \geqslant 3 !{ }^{3} \mathrm{D}_{i k j ; i k j} \geqslant 0 \text {. }
$$

If the $3-\mathrm{RDM}$ is not known, then one may take

$$
\left({ }^{1} \mathrm{D}_{i ; j}\right)^{2}{ }^{1} \mathrm{D}_{k ; k}+2 !{ }^{2} \mathrm{D}_{i k ; i k} \geqslant 22 !{ }^{2} \mathrm{D}_{i k ; j k}{ }^{1} \mathrm{D}_{i ; j}
$$

as the highest upper bound for this class of 2-RDM offdiagonal elements.

This is an important condition but it clearly does not guarantee that condition (31) is fulfilled. It must therefore be emphasized that the dependence on the 3-RDM diagonal element (and vice versa) cannot strictly be removed.

On the other hand, it must be stressed that Eq. (31) should be imposed as a limiting condition when constructing the 3-RDM from the 1- and 2-RDM's during the it-CSE. At this stage, let us point out that different ways of permuting the indices generate other different but equivalent inequalities. Also, combinations of equivalent inequalities may give rise to other relations. However, the inequalities just reported describe, in our view, the main limiting conditions for this type of 2-RDM off-diagonal elements.

Although we are primarily interested here on the 2-RDM $N$-representability conditions, we wish to report another result which sets up another important condition to the 3-G off-diagonal elements and is directly involved in the 3-RDM construction during the it-CSE. Thus, through the use of similar algebra operations as above, it may be shown that

$$
\begin{aligned}
{ }^{2} G_{i, p ; q, r} & =\sum_{\mathcal{L}^{\prime} \neq \mathcal{L}}\left\langle\mathcal{L}\left|\mathrm{b}_{i}^{\dagger} \mathrm{b}_{p}\right| \mathcal{L}^{\prime}\right\rangle\left\langle\mathcal{L}^{\prime}\left|\mathrm{b}_{r}^{\dagger} \mathrm{b}_{q}\right| \mathcal{L}\right\rangle \\
& =\sum_{\mathcal{L}^{\prime} \neq \mathcal{L}}\left\langle\mathcal{L}\left|\mathrm{b}_{p} \mathrm{~b}_{i}^{\dagger}\right| \mathcal{L}^{\prime}\right\rangle\left\langle\mathcal{L}^{\prime}\left|\mathrm{b}_{q} \mathrm{~b}_{r}^{\dagger}\right| \mathcal{L}\right\rangle={ }^{2} \bar{G}_{i, p ; q, r}
\end{aligned}
$$

and

$$
{ }^{3} G_{i, j, p ; r, s, q}+{ }^{3} \bar{G}_{i, j, p ; r, s, q}=\delta_{p, q}{ }^{2} G_{i, j ; r, s} .
$$

The bar denotes that the transition density matrices involved are HRDM's. The previous relation, jointly with the expres- 
sions for the diagonal elements of the 3-RDM and 3-HRDM, which are positive, allow us to deduce the relation

$$
\begin{aligned}
& { }^{1} \overline{\mathrm{D}}_{i ; i}{ }^{1} \overline{\mathrm{D}}_{j ; j}{ }^{1} \overline{\mathrm{D}}_{k ; k}+{ }^{1} \overline{\mathrm{D}}_{i ; i}{ }^{2} G_{j, j ; k, k}+{ }^{1} \overline{\mathrm{D}}_{j ; j}{ }^{2} G_{i, i ; k, k}+{ }^{2} G_{i, i ; j, j} \\
& \quad \geqslant{ }^{3} G_{i, i, k ; j, j, k} .
\end{aligned}
$$

\section{2-RDM off-diagonal elements with four different indices}

In order to find bounds for the 2-RDM off-diagonal elements with four different indices one must consider the 4- $G$ and 4-RDM diagonal elements. The pure four-body correlation term coincides with one of the three 4- $G$ positive matrices that can be defined by extending the argument used in the lower order cases. A general element of this matrix is

$$
\begin{aligned}
{ }^{4} G_{i, j, k, l ; p, q, r, s}= & \sum_{\mathcal{L}^{\prime}, \mathcal{L}^{\prime \prime}, \mathcal{L}^{\prime \prime \prime} \neq \mathcal{L}}\left\langle\mathcal{L}\left|\mathrm{b}_{i}^{\dagger} \mathrm{b}_{j}\right| \mathcal{L}^{\prime}\right\rangle\left\langle\mathcal{L}^{\prime}\left|\mathrm{b}_{k}^{\dagger} \mathrm{b}_{l}\right| \mathcal{L}^{\prime \prime}\right\rangle \\
& \times\left\langle\mathcal{L}^{\prime \prime}\left|\mathrm{b}_{s}^{\dagger} \mathrm{b}_{r}\right| \mathcal{L}^{\prime \prime \prime}\right\rangle\left\langle\mathcal{L}^{\prime \prime \prime}\left|\mathrm{b}_{q}^{\dagger} \mathrm{b}_{p}\right| \mathcal{L}\right\rangle .
\end{aligned}
$$

Developing the positive diagonal element of this matrix in a similar way as for the $3-G$ and remembering that

$$
{ }^{2} G_{i, j ; l, k}=2 !{ }^{2} \mathrm{D}_{i k ; j l}-{ }^{1} \mathrm{D}_{i ; j}{ }^{1} \mathrm{D}_{k ; l} \quad(j \neq k)
$$

one has:

$$
\begin{aligned}
& \left.\begin{array}{c}
2\left(-2 !^{2} \mathrm{D}_{i k ; j k}+3 !^{3} \mathrm{D}_{i k l ; j k l}\right){ }^{1} \mathrm{D}_{i ; j} \\
+\left({ }^{1} \mathrm{D}_{i ; j}\right){ }^{2} \mathrm{D}_{k ; k}+2 !^{2} \mathrm{D}_{i k ; i k} \\
+4 !^{4} \mathrm{D}_{i k l j ; i k l j}-\left({ }^{2} G_{i, j ; l, k}\right)^{2}
\end{array}\right\} \\
& \geqslant 3 !{ }^{3} D_{i k j ; i k j}+3 !{ }^{3} \mathrm{D}_{i k l ; i k l}+2 !\left({ }^{1} \mathrm{D}_{i ; j}\right)^{22} \mathrm{D}_{k l ; k l} \geqslant 0 \text {. }
\end{aligned}
$$

The element ${ }^{2} G_{i, j ; l, k}$ is the two-body part of ${ }^{2} \mathrm{D}_{i k ; j l}$ and, as can be seen, it is not only interdependent with the diagonal 4-RDM but also depends on the ${ }^{3} \mathrm{D}_{i k l ; j k l}$ element. Thus, as in the previous case, the interdependence among this class of off-diagonal elements and the higher-order RDM elements cannot be removed. As above, this inequality may be added to those derived from the positivity of the 4-RDM and 4-HRDM diagonal elements but, since the reasoning follows the same pattern, it is not included here. However, it must be remembered that these constraints should be satisfied when constructing 4-RDM diagonal elements which simultaneously involve several frontier orbitals. These results will be further discussed in Sec. VII.

\section{Second-order pseudoidempotency relation}

Let us consider the diagonal element of another positive ${ }^{4} G$ matrix:

$$
\begin{aligned}
0 \leqslant & \sum_{\mathcal{L}^{\prime} \neq \mathcal{L}}\left\langle\mathcal{L}\left|\mathrm{b}_{i}^{\dagger} \mathrm{b}_{j}^{\dagger} \mathrm{b}_{l} \mathrm{~b}_{m}\right| \mathcal{L}^{\prime}\right\rangle\left\langle\mathcal{L}^{\prime}\left|\mathrm{b}_{m}^{\dagger} \mathrm{b}_{l}^{\dagger} \mathrm{b}_{j} \mathrm{~b}_{i}\right| \mathcal{L}\right\rangle \\
= & \left\langle\mathcal{L}\left|\mathrm{b}_{i}^{\dagger} \mathrm{b}_{j}^{\dagger} \mathrm{b}_{l} \mathrm{~b}_{m} \mathrm{~b}_{m}^{\dagger} \mathrm{b}_{l}^{\dagger} \mathrm{b}_{j} \mathrm{~b}_{i}\right| \mathcal{L}\right\rangle-\left\langle\mathcal{L}\left|\mathrm{b}_{i}^{\dagger} \mathrm{b}_{j}^{\dagger} \mathrm{b}_{l} \mathrm{~b}_{m}\right| \mathcal{L}\right\rangle \\
& \times\left\langle\mathcal{L}\left|\mathrm{b}_{m}^{\dagger} \mathrm{b}_{l}^{\dagger} \mathrm{b}_{j} \mathrm{~b}_{i}\right| \mathcal{L}\right\rangle
\end{aligned}
$$

and add it up over the $m$ and $l$ indices:

$$
\left(\begin{array}{c}
2 K-N+2 \\
2
\end{array}\right) 2 !^{2} \mathrm{D}_{i j ; i j}-\left(2 !^{2} \mathrm{D}\right)_{i j ; i j}^{2} \geqslant 0 \text {. }
$$

The idempotency of the density matrix ( $N$-RDM) is thus replaced by relation (39), which links the diagonal elements of the 2-RDM and of its square matrix.

\section{MATRIX EXPRESSION OF THE WAVE-FUNCTION ANTISYMMETRY AND THE FERMION RELATIONS}

\section{A. Matrix expression of the $N$-electron wave function antisymmetry}

A general function $\mathcal{L}$ describing a normalized $N$-electron state

$$
|\mathcal{L}\rangle={ }^{N} \mathrm{~B}_{\mathcal{L}}^{\dagger}|0\rangle
$$

may be developed in the $N$-electron determinant basis. Therefore,

$$
{ }^{N} \mathrm{~B}_{\mathcal{L}}^{\dagger}=\sum_{\Lambda}\langle\Lambda \mid \mathcal{L}\rangle{ }^{N} \mathrm{~B}_{\Lambda}^{\dagger}
$$

where ${ }^{N} \mathrm{~B}_{\mathcal{L}}^{\dagger}$ is a linear combination of the basic global operators ${ }^{N} \mathrm{~B}_{\Lambda}^{\dagger}$ defined previously. The algebra of these operators is studied in Appendix B.

The properties of ${ }^{N} \mathrm{~B}_{\mathcal{L}}^{\dagger}$ and ${ }^{N} \mathrm{~B}_{\mathcal{L}}$, reflecting the antisymmetry and normalization of $|\mathcal{L}\rangle$ are expressed by their commutator (anticommutator) for $N$ even (odd). A generic element of the matrix representation of this commutator/ anticommutator is

$$
\begin{aligned}
\left\langle\Pi\left|\left[{ }^{N} \mathrm{~B}_{\mathcal{L}},{ }^{N} \mathrm{~B}_{\mathcal{L}}^{\dagger}\right]_{\mp}\right| \Gamma\right\rangle= & \sum_{\Lambda, \Omega}\langle\Lambda \mid \mathcal{L}\rangle\langle\mathcal{L} \mid \Omega\rangle \\
& \times\left\langle\Pi\left|\left[{ }^{N} \mathrm{~B}_{\Omega},{ }^{N} \mathrm{~B}_{\Lambda}^{\dagger}\right]_{\mp}\right| \Gamma\right\rangle \\
\equiv & \sum_{\Lambda, \Omega}^{N} \mathrm{D}_{\Lambda, \Omega}^{\mathcal{L}}\left\langle\Pi\left|\left[{ }^{N} \mathrm{~B}_{\Omega},{ }^{N} \mathrm{~B}_{\Lambda}^{\dagger}\right] \mp\right| \Gamma\right\rangle .
\end{aligned}
$$

Remembering that the bra and ket states, as well as the operators refer to $N$ electrons, one may write

$$
\left\langle\Pi\left|{ }^{N} \mathrm{~B}_{\Omega}{ }^{N} \mathrm{~B}_{\Lambda}^{\dagger}\right| \Gamma\right\rangle \equiv\left\langle\Omega\left|{ }^{N} \mathrm{~B}_{\Pi}{ }^{N} \mathrm{~B}_{\Gamma}^{\dagger}\right| \Lambda\right\rangle
$$

and

$$
\left\langle\Pi\left|{ }^{N} \mathrm{~B}_{\Lambda}^{\dagger}{ }^{N} \mathrm{~B}_{\Omega}\right| \Gamma\right\rangle \equiv \delta_{\Lambda, \Pi} \delta_{\Omega, \Gamma} \equiv\left\langle\Omega\left|{ }^{N} \mathrm{~B}_{\Gamma}^{\dagger}{ }^{N} \mathrm{~B}_{\Pi}\right| \Lambda\right\rangle
$$

and then one has

$$
\begin{aligned}
& \left\langle\Pi\left|\left[{ }^{N} \mathrm{~B}_{\mathcal{L}},{ }^{N} \mathrm{~B}_{\mathcal{L}}^{\dagger}\right]_{\mp}\right| \Gamma\right\rangle \\
& \equiv \sum_{\Lambda, \Omega}^{N} \mathrm{D}_{\Lambda, \Omega}^{\mathcal{L}}\left\langle\Omega\left|\left[{ }^{N} \mathrm{~B}_{\Pi},{ }^{N} \mathrm{~B}_{\Gamma}^{\dagger}\right]_{\mp}\right| \Lambda\right\rangle \\
& \equiv\left\langle\mathcal{L}\left|\left[{ }^{N} \mathrm{~B}_{\Pi},{ }^{N} \mathrm{~B}_{\Gamma}^{\dagger}\right]_{\mp}\right| \mathcal{L}\right\rangle
\end{aligned}
$$


In Sec. V B the explicit development of the right-hand side of Eq. (45), which is the mathematical matrix expression of the antisymmetry and normalization of the $N$-electron wave function, will be carried out.

\section{B. Fermion relations}

The expectation value of the basic fermion anticommutation relation gives

$$
\left\langle\mathcal{L}\left|\mathrm{b}_{j} \mathrm{~b}_{i}^{\dagger}\right| \mathcal{L}\right\rangle+\left\langle\mathcal{L}\left|\mathrm{b}_{i}^{\dagger} \mathrm{b}_{j}\right| \mathcal{L}\right\rangle=\delta_{i j} \equiv{ }^{1} \overline{\mathrm{D}}_{i j}+{ }^{1} \mathrm{D}_{i j} .
$$

This is the first of a series of equations relating the RDM's and HRDM's which will hereafter be denoted generically as fermion relations (FR). Therefore, for $i=j$, since both the 1-RDM and 1-HRDM are positive semidefinite matrices, the maximum value that this diagonal element can take is 1 . Since it is easy to show that the $1-\mathrm{RDM}$ and 1-HRDM commute and can be simultaneously diagonalized, formula (46) expresses the well-known necessary and sufficient ensemble $N$-representability condition for the 1-RDM [5].

By taking the trace of relation (46), one obtains what can be considered the 0-FR

$$
\operatorname{tr}^{1} \overline{\mathbf{D}}+\operatorname{tr}^{1} \mathbf{D}=(2 K-N)+N=2 K .
$$

The commutation relation for pairs of fermion operators in normal form is

$$
\begin{aligned}
{\left[\mathrm{b}_{l} \mathrm{~b}_{k}, \mathrm{~b}_{i}^{\dagger} \mathrm{b}_{j}^{\dagger}\right]_{-}=} & \delta_{l j} \delta_{k i}-\delta_{l i} \delta_{k j}-\delta_{l j} \mathrm{~b}_{i}^{\dagger} \mathrm{b}_{k}-\delta_{i k} \mathrm{~b}_{j}^{\dagger} \mathrm{b}_{l}+\delta_{l i} \mathrm{~b}_{j}^{\dagger} \mathrm{b}_{k} \\
& +\delta_{k j} \mathrm{~b}_{i}^{\dagger} \mathrm{b}_{l},
\end{aligned}
$$

and with

$$
\begin{gathered}
|\lambda\rangle=|i j\rangle, \quad(i<j), \quad|\gamma\rangle=|k l\rangle, \quad(k<l), \\
{\left[\mathrm{b}_{l} \mathrm{~b}_{k}, \mathrm{~b}_{i}^{\dagger} \mathrm{b}_{j}^{\dagger}\right]_{-}=\left[{ }^{2} \mathrm{~B}_{\gamma},{ }^{2} \mathrm{~B}_{\lambda}^{\dagger}\right]_{-}=\delta_{\lambda \gamma}-\sum_{t v}^{2 K}\left\langle\lambda\left|\mathrm{b}_{t}^{\dagger} \mathrm{b}_{v}\right| \gamma\right\rangle \mathrm{b}_{t}^{\dagger} \mathrm{b}_{v} .}
\end{gathered}
$$

The four terms of the right-hand side of Eq. (48), involving a single Kronecker delta have been gathered in the last term of Eq. (50) by applying the arguments described in Appendix A and by introducing the auxiliary indices $t$ and $v$, which obviously do not contribute except when they are respectively $(l, j)$, or $(i, k)$, or $(l, i)$, or $(j, k)$.

Let us now take the expectation value of this commutator:

$$
\left\langle\mathcal{L}\left|\left[{ }^{2} \mathrm{~B}_{\gamma},{ }^{2} \mathrm{~B}_{\lambda}^{\dagger}\right]_{-}\right| \mathcal{L}\right\rangle=\delta_{\lambda \gamma}-\sum_{t v}^{2 K}\left\langle\lambda\left|\mathrm{b}_{t}^{\dagger} \mathrm{b}_{v}\right| \gamma\right\rangle\left\langle\mathcal{L}\left|b_{t}^{\dagger} \mathrm{b}_{v}\right| \mathcal{L}\right\rangle .
$$

That is,

$$
{ }^{2} \overline{\mathrm{D}}_{\lambda ; \gamma}-{ }^{2} D_{\lambda ; \gamma}=\delta_{\lambda \gamma}-\operatorname{tr}\left({ }^{1} \mathbf{D}^{\lambda \gamma}{ }^{1} \mathbf{D}\right) .
$$

This is the 2-FR which coincides with the well known $\mathbf{Q}$ $N$-representability condition [10].
We have obtained the 3-FR, 4-FR and 5-FR cases, and it can be inferred that the general expression for the $N$-FR is $[47,48]$.

$$
\begin{aligned}
{ }^{N} \bar{D}_{\Lambda \Omega}-(-1)^{N}{ }^{N} \mathrm{D}_{\Lambda \Omega}= & \delta_{\Lambda \Omega}-\operatorname{tr}\left({ }^{1} \mathbf{D}^{\Lambda \Omega}{ }^{1} \mathbf{D}\right) \\
& +\operatorname{tr}\left({ }^{2} \mathbf{D}^{\Lambda \Omega}{ }^{2} \mathbf{D}\right)-\operatorname{tr}\left({ }^{3} \mathbf{D}^{\Lambda \Omega}{ }^{3} \mathbf{D}\right)+\cdots \\
& +(-1)^{(N-1)} \operatorname{tr}\left({ }^{(N-1)} \mathbf{D}^{\Lambda \Omega}{ }^{(N-1)} \mathbf{D}\right) .
\end{aligned}
$$

That is,

$$
{ }^{N} \overline{\mathrm{D}}_{\Lambda \Omega}-(-1)^{N}{ }^{N} \mathrm{D}_{\Lambda \Omega}=\sum_{q=0}^{N-1}(-1)^{q} \operatorname{tr}\left({ }^{q} \mathbf{D}^{\Lambda \Omega q} \mathbf{D}\right) .
$$

Clearly, this equation is the development of Eq. (45) which describes the normalization and antisymmetry of the $\mathrm{N}$-electron wave function.

A similar set of relations may be written by interchanging the roles of particles and holes. This equivalence is exploited to obtain the constructing algorithm for the 3- and 4-RDM's [30].

It should be emphasized that these, like other $\mathrm{N}$-representability conditions so far mentioned, do not involve a single RDM, but a relation among elements of RDM's of several orders. That is, expression (54) adds to the generally known properties the information on how ${ }^{N} \mathbf{D}$ and ${ }^{N} \overline{\mathbf{D}}$ are uniquely related to the expansion of the lower-order RDM's, since Eq. (54) may be rewritten as

$$
{ }^{N} \overline{\mathbf{D}}-(-1)^{N}{ }^{N} \mathbf{D}=\sum_{q=0}^{N-1}(-1)^{q} \Gamma_{q}^{N q} \mathbf{D}
$$

where $\Gamma_{q}^{N}$ represents an expansion mapping $[11,12]$. Here $\Gamma_{q}^{N}$ maps the matrix ${ }^{q} \mathbf{D}$ into another matrix represented in the $N$-electron space, whose $\{\Lambda \Omega\}$ element is $\operatorname{tr}\left({ }^{q} \mathbf{D}^{\Lambda \Omega q} \mathbf{D}\right)$, which is not an RDM nor a density matrix [its trace is equal to $\left.\left(\begin{array}{l}N \\ q\end{array}\right)\left(\begin{array}{c}2 K-q \\ N-q\end{array}\right)\right]$.

\section{CONTRACTION OF THE $N$-FR}

In Sec. $\mathrm{V}$ we saw that the $N$-FR [Eq. (54) or (55)] expresses in matrix form all the properties of the $N$-electron wave functions. Moreover, Eq. (54) is a relation among RDM's of all orders. Therefore, by applying contracting mapping (CM) $[42,43,47,48]$ (see Appendix B 1) to any order $p$ to both sides of Eq. (54), one should obtain, by construction, an $N$-representable relation. That is, the resulting relation has, as a whole, a preimage relation in the antisymmetric $\mathrm{N}$-electron space. Consequently, the matrices, representing density operators and interlinked through the result of this contraction, should be $N$ representable.

This rather long section describes the main algebraic steps involved in the contraction of the N-FR [Eq. (54)]. We consider that this demonstration, in itself, constitutes a good example of the rich RDM methodology, nevertheless, since its reading may be rather involved, the nonspecialist may 
proceed to the last paragraphs of the section where we comment on the result.

Let us proceed now to contract the $N$-FR by applying the CM (see Appendix B)

$$
\begin{aligned}
\sum_{\Lambda \Omega}{ }^{p} \mathrm{D}_{\alpha \beta}^{\Lambda \Omega}\left({ }^{N} \overline{\mathbf{D}}_{\Lambda \Omega}-(-1)^{N}{ }^{N} \mathrm{D}_{\Lambda \Omega}\right) \\
=\sum_{\Lambda \Omega}{ }^{p} \mathrm{D}_{\alpha \beta}^{\Lambda \Omega}\left\{\begin{array}{l}
\delta_{\Lambda \Omega}-\operatorname{tr}\left({ }^{1} \mathbf{D}^{\Lambda \Omega 1} \mathbf{D}\right) \\
+\operatorname{tr}\left({ }^{2} \mathbf{D}^{\Lambda \Omega}{ }^{2} \mathbf{D}\right) \\
-\operatorname{tr}\left({ }^{3} \mathbf{D}^{\Lambda \Omega}{ }^{3} \mathbf{D}\right)+\ldots \\
+(-1)^{(N-1)} \operatorname{tr}\left({ }^{(N-1)} \mathbf{D}^{\Lambda \Omega N-1} \mathbf{D}\right)
\end{array}\right. \\
=\sum_{\Lambda \Omega}{ }^{p} \mathrm{D}_{\alpha \beta}^{\Lambda \Omega} \sum_{q=0}^{N-1}(-1)^{q} \operatorname{tr}\left({ }^{q} \mathbf{D}^{\Lambda \Omega q} \mathbf{D}\right),
\end{aligned}
$$

where $\alpha$ and $\beta$ are $p$-electron states of the Slater determinant type.

The left-hand side of this equation is the direct application of the $\mathrm{CM}$, and gives

$$
\left(\begin{array}{c}
2 K-(N+p) \\
N-p
\end{array}\right){ }^{p} \bar{D}_{\alpha \beta}-(-1)^{N p} \mathrm{D}_{\alpha \beta} .
$$

In what follows we will evaluate the right-hand side of Eq. (56).

\section{Evaluation of the right-hand side terms of relation (56)}

The algebra tools developed in the Appendixes allow us to solve the problem, although, as has been mentioned, the development is a rather long one. In what follows, we will represent the right-hand side of Eq. (56) by Z. Let us start by rewriting

$$
\begin{aligned}
Z= & \sum_{\Lambda \Omega}{ }^{p} \mathrm{D}_{\alpha \beta}^{\Lambda \Omega} \sum_{q=0}^{N-1}(-1)^{q} \operatorname{tr}\left({ }^{q} \mathbf{D}^{\Lambda \Omega q} \mathbf{D}\right) \\
= & \sum_{\Lambda \Omega} \sum_{q=0}^{N-1}(-1)^{q} \sum_{\eta, \nu}^{q}\left\langle\Lambda\left|{ }^{p} \mathrm{~B}_{\alpha}^{\dagger p} \mathrm{~B}_{\beta}\right| \Omega\right\rangle \\
& \times\left\langle\Omega\left|{ }^{q} \mathrm{~B}_{\eta}^{\dagger}{ }^{q} \mathrm{~B}_{\nu}\right| \Lambda\right\rangle^{q} \mathrm{D}_{\nu \eta},
\end{aligned}
$$

where we have taken into account that $\operatorname{tr}\left({ }^{q} \mathbf{D}^{\Lambda \Omega q} \mathbf{D}\right)$ $=\operatorname{tr}\left({ }^{q} \mathbf{D}^{\Omega \Lambda q} \mathbf{D}\right)$. After summing over $\Omega$ we will decompose the right-hand side of this equation into

$$
\begin{aligned}
Z= & \sum_{\Lambda} \sum_{q=0}^{p}(-1)^{q} \sum_{\eta, \nu}^{\left(\begin{array}{c}
2 K \\
q
\end{array}\right)}\left\langle\Lambda\left|{ }^{p} \mathrm{~B}_{\alpha}^{\dagger p} \mathrm{~B}_{\beta}{ }^{q} \mathrm{~B}_{\eta}^{\dagger}{ }^{q} \mathrm{~B}_{\nu}\right| \Lambda\right\rangle^{q} \mathrm{D}_{\nu \eta} \\
& +\sum_{\Lambda} \sum_{q=p+1}^{N-1}(-1)^{q} \sum_{\eta, \nu}\left\langle\Lambda\left|{ }^{p} \mathrm{~B}_{\alpha}^{\dagger p} \mathrm{~B}_{\beta}{ }^{q} \mathrm{~B}_{\eta}^{\dagger}{ }^{q} \mathrm{~B}_{\nu}\right| \Lambda\right\rangle^{q} \mathrm{D}_{\nu \eta} .
\end{aligned}
$$

Here we will only report in detail how to evaluate the part $q>p$ (hereafter denoted by X) since for $p>q$ all the steps are very similar. Let us therefore replace ${ }^{p} \mathrm{~B}_{\beta}{ }^{q} \mathbf{B}_{\eta}^{\dagger}$ by its normal form according to expression (B11), we have

$$
\begin{aligned}
& X=\sum_{\Lambda} \sum_{q=p+1}^{N-1}(-1)^{q} \sum_{\eta, \nu}^{\left(\begin{array}{c}
2 K \\
2
\end{array}\right)} \sum_{k=0}^{p}(-1)^{(q-p) p+k}
\end{aligned}
$$

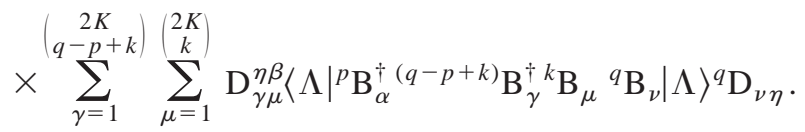

We now apply the same arguments as in relations (B14) and (B16) of the Appendix B:

$$
\begin{aligned}
& X=\sum_{q=p+1}^{N-1} \sum_{\eta, \nu}^{\left(\begin{array}{c}
2 K \\
q
\end{array}\right)} \sum_{k=0}^{p} \sum_{\gamma=1}^{\left(\begin{array}{c}
2 K \\
q-p+k
\end{array}\right)} \sum_{\mu=1}^{\left(\begin{array}{c}
2 K \\
k
\end{array}\right)}(-1)^{q}(-1)^{(q-p) p+k}, \\
& \times \mathrm{D}_{\gamma \mu}^{\eta \beta q} \mathrm{D}_{\nu \eta}\langle\mu \nu \mid \alpha \gamma\rangle\left(\begin{array}{c}
2 K-q-k \\
N-q-k
\end{array}\right) \\
& =\sum_{q=p+1}^{N-1} \sum_{\eta, \nu}^{\left(\begin{array}{c}
2 K \\
q
\end{array}\right)} \sum_{k=0}^{p}(-1)^{(q-p) p+k}
\end{aligned}
$$

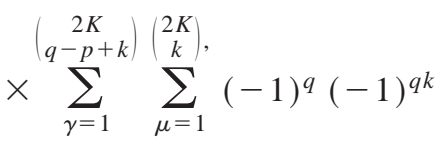

$$
\begin{aligned}
& \times(-1)^{(q-p+k) p}{ }^{q} \mathrm{D}_{\nu \eta} \mathrm{D}_{\gamma \mu}^{\eta \beta}\left\langle\left.\nu\right|^{k} \mathrm{~B}_{\mu}{ }^{(q-p+k)} \mathrm{B}_{\gamma}^{\dagger} \mid \alpha\right\rangle \\
& \times\left(\begin{array}{c}
2 K-q-k \\
N-q-k
\end{array}\right) .
\end{aligned}
$$

Let us focus our attention, for the time being, on the part which must now be transformed:

$$
\left(\sum_{\gamma=1}^{\left(\begin{array}{c}
2 K \\
q-p+k
\end{array}\right)} \sum_{\mu=1}^{\left(\begin{array}{c}
2 K \\
k
\end{array}\right)} \mathrm{D}_{\gamma \mu}^{\eta \beta}\left\langle\left.\nu\right|^{k} \mathrm{~B}_{\mu}{ }^{(q-p+k)} \mathrm{B}_{\gamma}^{\dagger} \mid \alpha\right\rangle .\right.
$$

By again applying formula (B11) (it is the only possibility consistent with our assumption $q>p$ ) in order to transform ${ }^{k} \mathrm{~B}_{\mu}^{(q-p+k)} \mathrm{B}_{\gamma}^{\dagger}$ into its normal form, one has

$$
\begin{aligned}
& \left(\begin{array}{c}
2 k \\
q-p+k
\end{array}\right)\left(\begin{array}{c}
2 K \\
k
\end{array}\right) \quad k \quad\left(\begin{array}{c}
2 K \\
q-p+r
\end{array}\right)\left(\begin{array}{c}
2 K \\
r
\end{array}\right) \\
& \sum_{\gamma=1} \sum_{\mu=1} \sum_{r=0} \sum_{\tau=1} \sum_{\omega=1} \\
& \times(-1)^{(q-p) k+r} \mathrm{D}_{\gamma \mu}^{\eta \beta}\left\langle\left.\nu\right|^{(q-p+r)} \mathrm{B}_{\tau}^{\dagger r} \mathrm{~B}_{\omega} \mid \alpha\right\rangle \mathrm{D}_{\tau \omega}^{\gamma \mu} .
\end{aligned}
$$

Now let us write $|\gamma\rangle \equiv|\tau x\rangle s_{1}$ and $|\mu\rangle \equiv|\omega x\rangle s_{2}$ where $s_{1}$ and $s_{2}$ are the corresponding signs. We will now replace these forms of $|\gamma\rangle$ and $|\mu\rangle$ into Eq. (60) and summing up over the new index $x$ (instead than over $\gamma$ and $\mu$ ), one has: 


$$
\begin{aligned}
\sum_{x=1}^{\left(\begin{array}{c}
2 K \\
k-r
\end{array}\right)} \sum_{r=0}^{k} \sum_{\tau=1}^{\left(\begin{array}{c}
2 K \\
q-p+r
\end{array}\right)} \sum_{\omega=1}^{\left(\begin{array}{c}
2 K \\
r
\end{array}\right)}(-1)^{(q-p) k+r}\left\langle\left.\nu\right|^{(q-p+r)} \mathrm{B}_{\tau}^{\dagger \dagger} \mathrm{B}_{\omega} \mid \alpha\right\rangle \\
\quad \times\left\langle\left.\eta\right|^{(q-p+r)} \mathrm{B}_{\tau}^{\dagger(k-r)} \mathrm{B}_{x}^{\dagger(k-r)} \mathrm{B}_{x}{ }^{r} \mathrm{~B}_{\omega} \mid \beta\right\rangle \\
=\sum_{r=0} \sum_{\tau=1} \sum_{\omega=1}^{\left(\begin{array}{c}
2 K \\
q-p+r
\end{array}\right)}(-1)^{\left(\begin{array}{c}
2 K \\
r
\end{array}\right)} \\
\quad \times\left\langle\left.\nu\right|^{(q-p+r) k+r}\left(\begin{array}{l}
p-r \\
k-r
\end{array}\right)\right.
\end{aligned}
$$

Now we may apply the arguments leading to relation (B19) and find

$$
\sum_{r=0}^{k}(-1)^{(q-p) k+r}\left(\begin{array}{l}
p-r \\
k-r
\end{array}\right) \operatorname{tr}\left({ }^{(p-r)} \mathbf{D}^{\nu \eta}(p-r) \mathbf{D}^{\alpha \beta}\right)
$$

This partial result now inserts Eq. (62) into Eq. (61), giving

$$
\begin{aligned}
X= & \sum_{q=p+1}^{N-1}(-1)^{q} \sum_{\eta, \nu} \sum_{k=0}^{p}(-1)^{(q-p) p+k} \sum_{r=0}^{k}\left(\begin{array}{c}
p-r \\
q-r
\end{array}\right) \\
& \times(-1)^{(q-p) k+r}(-1)^{q k} \\
& \times(-1)^{(q-p+k) p} \quad{ }^{q} \mathrm{D}_{\nu \eta} \operatorname{tr}\left({ }^{(p-r)} \mathbf{D}^{\nu \eta(p-r)} \mathbf{D}^{\alpha \beta}\right) \\
& \times\left(\begin{array}{c}
2 K-q-k \\
N-q-k
\end{array}\right) .
\end{aligned}
$$

When summing now over $\eta$ and $\nu$, one has the final result for the case $q>p$ in Eq. (61):

$$
\begin{aligned}
X= & \sum_{q=p+1}^{N-1} \sum_{k=0}^{p} \sum_{r=0}^{k}(-1)^{(q+k+r)}\left(\begin{array}{c}
p-r \\
k-r
\end{array}\right) \operatorname{tr}\left({ }^{(p-r)} \mathbf{D}^{(p-r)} \mathbf{D}^{\alpha \beta}\right) \\
& \times\left(\begin{array}{c}
N-p+r \\
N-q
\end{array}\right)\left(\begin{array}{c}
2 K-q-k \\
N-q-k
\end{array}\right) .
\end{aligned}
$$

The result following from the part of expression (59) with $p<q($ called $Y$ ) gives

$$
\begin{aligned}
Y= & \sum_{q^{\prime}=0}^{p} \sum_{k^{\prime}=0}^{q^{\prime}} \sum_{r^{\prime}=0}^{k}(-1)^{\left(q+k^{\prime}+r^{\prime}\right)}\left(\begin{array}{c}
q^{\prime}-r^{\prime} \\
k^{\prime}-r^{\prime}
\end{array}\right) \\
& \times\left(\begin{array}{c}
2 K-p-k^{\prime} \\
N-p-k^{\prime}
\end{array}\right) \operatorname{tr}\left({ }^{\left(q^{\prime}-r^{\prime}\right)} \mathbf{D}^{\left(q^{\prime}-r^{\prime}\right)} \mathbf{D}^{\alpha \beta}\right)\left(\begin{array}{c}
N-q^{\prime}+r^{\prime} \\
N-q^{\prime}
\end{array}\right) .
\end{aligned}
$$

Now the contributions from $q>p$ and from $q<p$ must be summed up:

$$
\begin{aligned}
Z= & \sum_{q^{\prime}=0}^{p} \sum_{k^{\prime}=0}^{q^{\prime}} \sum_{r^{\prime}=0}^{k^{\prime}}(-1)^{\left(q^{\prime}+k^{\prime}+r^{\prime}\right)}\left(\begin{array}{c}
q^{\prime}-r^{\prime} \\
k^{\prime}-r^{\prime}
\end{array}\right) \\
& \times\left(\begin{array}{c}
2 K-p-k^{\prime} \\
N-p-k^{\prime}
\end{array}\right) \operatorname{tr}\left({ }^{\left(q^{\prime}-r^{\prime}\right)} \mathbf{D}^{\left(q^{\prime}-r^{\prime}\right)} \mathbf{D}^{\alpha \beta}\right)\left(\begin{array}{c}
N-q^{\prime}+r^{\prime} \\
N-q^{\prime}
\end{array}\right) \\
& +\sum_{q=p+1} \sum_{k=0}^{p} \sum_{r=0}^{k}(-1)^{(q+k+r)}\left(\begin{array}{c}
p-r \\
k-r
\end{array}\right) \\
& \times\left(\begin{array}{c}
2 K-q-k \\
N-q-k
\end{array}\right) \operatorname{tr}\left({ }^{(p-r)} \mathbf{D}^{(p-r)} \mathbf{D}^{\alpha \beta}\right)\left(\begin{array}{c}
N-p+r \\
N-q
\end{array}\right)
\end{aligned}
$$

In order to unite the sum over the $q, k$, and $r$ indices with that on the $q^{\prime}, k^{\prime}$, and $r^{\prime}$ ones, we start by defining the variables $t=p-r$ and $t^{\prime}=q^{\prime}-r^{\prime}$, and replace the sums over $r$ and $r^{\prime}$ by the corresponding new indices

$$
\begin{aligned}
& Z=\sum_{q^{\prime}=0}^{p} \sum_{k^{\prime}=0}^{q^{\prime}} \sum_{t^{\prime}=q^{\prime}-k^{\prime}}^{q^{\prime}}(-1)^{\left(k^{\prime}+t^{\prime}\right)}\left(\begin{array}{c}
t^{\prime} \\
k^{\prime}-q^{\prime}+t^{\prime}
\end{array}\right) \\
& \times\left(\begin{array}{c}
2 K-p-k^{\prime} \\
N-p-k^{\prime}
\end{array}\right) \operatorname{tr}\left({ }^{t^{\prime}} \mathbf{D}^{t^{\prime}} \mathbf{D}^{\alpha \beta}\right)\left(\begin{array}{c}
N-t^{\prime} \\
N-q^{\prime}
\end{array}\right) \\
& +\sum_{q=p+1}^{N-1} \sum_{k=0}^{p} \sum_{t=p-k}^{p} \\
& \times(-1)^{(q+k+p-t)}\left(\begin{array}{c}
t \\
k-p+t
\end{array}\right) \operatorname{tr}\left({ }^{t} \mathbf{D}^{t} \mathbf{D}^{\alpha \beta}\right)\left(\begin{array}{c}
N-t \\
N-q
\end{array}\right) \\
& \times\left(\begin{array}{c}
2 K-q-k \\
N-q-k
\end{array}\right)
\end{aligned}
$$

By handling the limits of the sums one may write

$$
\begin{aligned}
Z= & \sum_{q^{\prime}=0}^{p} \sum_{t^{\prime}=0}^{q^{\prime}}(-1)^{t^{\prime}} \operatorname{tr}\left({ }^{t^{\prime}} \mathbf{D}^{t^{\prime}} \mathbf{D}^{\alpha \beta}\right)\left(\begin{array}{c}
N-t^{\prime} \\
N-q^{\prime}
\end{array}\right) \\
& \times \sum_{k^{\prime}=q^{\prime}-t^{\prime}}^{q^{\prime}}(-1)^{k^{\prime}}\left(\begin{array}{c}
t^{\prime} \\
k^{\prime}-q^{\prime}+t^{\prime}
\end{array}\right)\left(\begin{array}{c}
2 K-p-k^{\prime} \\
N-p-k^{\prime}
\end{array}\right) \\
& +\sum_{q=p+1}^{N-1} \sum_{t=0}^{p}(-1)^{(q+p-t)} \operatorname{tr}\left({ }^{t} \mathbf{D}^{t} \mathbf{D}^{\alpha \beta}\right) \\
& \times\left(\begin{array}{c}
N-t \\
N-q
\end{array}\right) \sum_{k=p-t}^{p}\left(\begin{array}{c}
t \\
k-p+t
\end{array}\right)(-1)^{k}\left(\begin{array}{c}
2 K-q-k \\
N-q-k
\end{array}\right) .
\end{aligned}
$$

The sums over $k$ and $k^{\prime}$ can be performed by applying, in a systematic way, the relation

$$
\left(\begin{array}{l}
A \\
B
\end{array}\right)-\left(\begin{array}{l}
A-1 \\
B-1
\end{array}\right)=\left(\begin{array}{c}
A-1 \\
B
\end{array}\right),
$$

or, in a more general way,

$$
\sum_{m=0}^{n}(-1)^{m}\left(\begin{array}{c}
n \\
m
\end{array}\right)\left(\begin{array}{c}
A-m \\
B-m
\end{array}\right)=\left(\begin{array}{c}
A-n \\
B
\end{array}\right) .
$$


one has

$$
\begin{aligned}
Z= & \sum_{q^{\prime}=0}^{p} \sum_{t^{\prime}=0}^{q^{\prime}}(-1)^{t^{\prime}} \operatorname{tr}\left({ }^{t^{\prime}} \mathbf{D}^{t^{\prime}} \mathbf{D}^{\alpha \beta}\right)(-1)^{q^{\prime}-t^{\prime}}\left(\begin{array}{c}
N-t^{\prime} \\
N-q^{\prime}
\end{array}\right) \\
& \times\left(\begin{array}{c}
2 K-p-q^{\prime} \\
N-p-q^{\prime}+t^{\prime}
\end{array}\right)+\sum_{q=p+1}^{N-1} \sum_{t=0}^{p}(-1)^{(q+p-t)} \operatorname{tr}\left({ }^{t} \mathbf{D}^{t} \mathbf{D}^{\alpha \beta}\right) \\
& \times(-1)^{p-t}\left(\begin{array}{c}
N-t \\
N-q
\end{array}\right)\left(\begin{array}{c}
2 K-q-p \\
N-q-p+t
\end{array}\right),
\end{aligned}
$$

that is,

$$
\begin{aligned}
Z= & \sum_{q^{\prime}=0}^{p} \sum_{t^{\prime}=0}^{q^{\prime}}(-1)^{q^{\prime}} \operatorname{tr}\left({ }^{t^{\prime}} \mathbf{D}^{t^{\prime}} \mathbf{D}^{\alpha \beta}\right)\left(\begin{array}{c}
N-t^{\prime} \\
N-q^{\prime}
\end{array}\right) \\
& \times\left(\begin{array}{c}
2 K-p-q^{\prime} \\
N-p-q^{\prime}+t^{\prime}
\end{array}\right)+\sum_{q=p+1}^{N-1} \sum_{t=0}^{p}(-1)^{q} \operatorname{tr}\left({ }^{t} \mathbf{D}^{t} \mathbf{D}^{\alpha \beta}\right) \\
& \times\left(\begin{array}{c}
N-t \\
N-q
\end{array}\right)\left(\begin{array}{c}
2 K-q-p \\
N-q-p+t
\end{array}\right) .
\end{aligned}
$$

Now the two series may be added up, and we have:

$$
Z=\sum_{t=0}^{p} \operatorname{tr}\left({ }^{t} \mathbf{D}^{t} \mathbf{D}^{\alpha \beta}\right) \sum_{q=t}^{N-1}(-1)^{q}\left(\begin{array}{c}
N-t \\
N-q
\end{array}\right)\left(\begin{array}{c}
2 K-p-q \\
N-p-q+t
\end{array}\right) .
$$

In order to perform the second sum, let us shift the index $q$ by $t$ and in Eq. (76) separate the term $t=p$.

$$
\begin{aligned}
Z= & \sum_{t=0}^{p-1}(-1)^{t} \operatorname{tr}\left({ }^{t} \mathbf{D}^{t} \mathbf{D}^{\alpha \beta}\right) \sum_{s=0}^{N-t-1}(-1)^{s}\left(\begin{array}{c}
N-t \\
s
\end{array}\right) \\
& \times\left(\begin{array}{c}
2 K-p-t-s \\
N-p-s
\end{array}\right)+(-1)^{p} \operatorname{tr}\left({ }^{p} \mathbf{D}^{p} \mathbf{D}^{\alpha \beta}\right) \\
& \times \sum_{s=0}^{N-p-1}(-1)^{s}\left(\begin{array}{c}
N-p \\
s
\end{array}\right)\left(\begin{array}{c}
2 K-2 p-s \\
N-p-s
\end{array}\right)
\end{aligned}
$$

Since binomial coefficients with negative lower index vanish, the only contributions that need to be considered arise from $s \leqslant N-p$. Therefore, it is possible to modify the upper limits of the sums in order to write in a very compact form:

$$
\begin{aligned}
Z= & \sum_{t=0}^{p-1}(-1)^{t} \operatorname{tr}\left({ }^{t} \mathbf{D}^{t} \mathbf{D}^{\alpha \beta}\right) \\
& \times \sum_{s=0}^{N-t}(-1)^{s}\left(\begin{array}{c}
N-t \\
s
\end{array}\right)\left(\begin{array}{c}
2 K-p-t-s \\
N-p-s
\end{array}\right) \\
& +(-1)^{p} \operatorname{tr}\left({ }^{p} \mathbf{D}^{p} \mathbf{D}^{\alpha \beta}\right)\left(\sum_{s=0}^{N-p}(-1)^{s}\left(\begin{array}{c}
N-p \\
s
\end{array}\right)\left(\begin{array}{c}
2 K-2 p-s \\
N-p-s
\end{array}\right)\right. \\
& \left.+(-1)^{(N-p)}\right),
\end{aligned}
$$

$$
\begin{aligned}
Z= & \sum_{t=0}^{p-1}(-1)^{t} \operatorname{tr}\left({ }^{t} \mathbf{D}^{t} \mathbf{D}^{\alpha \beta}\right)\left(\begin{array}{c}
2 K-p-N \\
N-p
\end{array}\right) \\
& +(-1)^{p}{ }^{p} \mathrm{D}_{\alpha \beta}\left(\begin{array}{c}
2 K-p-N \\
N-p
\end{array}\right)-(-1)^{N p} \mathrm{D}_{\alpha \beta} .
\end{aligned}
$$

Gathering now all the partial results just obtained, one has the final expression for Eq. (56)

$$
\begin{aligned}
& \left(\begin{array}{c}
2 K-N-p \\
N-p
\end{array}\right) p \overline{\mathrm{D}}_{\alpha \beta}-(-1)^{N p} \mathrm{D}_{\alpha \beta} \\
& =\sum_{t=0}^{p-1}(-1)^{t} \operatorname{tr}\left({ }^{t} \mathbf{D}^{t} \mathbf{D}^{\alpha \beta}\right)\left(\begin{array}{c}
2 K-p-N \\
N-p
\end{array}\right) \\
& \quad+(-1)^{p p} \mathrm{D}_{\alpha \beta}\left(\begin{array}{c}
2 K-p-N \\
N-p
\end{array}\right)-(-1)^{N p} \mathrm{D}_{\alpha \beta} .
\end{aligned}
$$

That is

$$
{ }^{p} \overline{\mathbf{D}}_{\alpha \beta}-(-1)^{p}{ }^{p} \mathbf{D}_{\alpha \beta}=\sum_{t=0}^{p-1}(-1)^{t} \operatorname{tr}\left({ }^{t} \mathbf{D}^{t} \mathbf{D}^{\alpha \beta}\right) .
$$

Relation (81) is the final result of the contraction of the $N$-FR and coincides with the $p$-FR which was obtained by taking the expectation value of the commutator $\left[{ }^{p} \mathrm{~B},{ }^{p} \mathrm{~B}^{\dagger}\right]_{ \pm}$. The contraction has been carried out from the $N$-FR to the $p$-FR. It could also have been done from any arbitrary $p$ to any arbitrary $q<p$.

The same result may be obtained in the Grassmann algebra formalism. The $p$-FR [36]:

$$
{ }^{p} \overline{\mathbf{D}}-(-1)^{p}{ }^{p} \mathbf{D}=\sum_{m=0}^{p-1}(-1)^{m}\left(\begin{array}{c}
p \\
m
\end{array}\right)\left(\mathbf{I}^{p-m} \wedge^{m} \mathbf{D}\right)\left({ }^{0} \mathbf{D} \equiv 1\right)
$$

contracts into the $(p-1)$-FR by means of the general relation:

$$
\begin{aligned}
& \mathbf{I}^{r} \wedge s \mathbf{D} \rightarrow s \frac{N-s+1}{(r+s)^{2}}\left(\mathbf{I}^{r} \bigwedge^{s-1} \mathbf{D}\right) \\
& +r \frac{2 K-r-2 s+1}{(r+s)^{2}}\left(\mathbf{I}^{r-1} \wedge^{s} \mathbf{D}\right) \text {. }
\end{aligned}
$$

From there, by recurrence, any $q$-FR $(q<p)$ may be reached. In that way, the usefulness of the algebra of transition RDM's, and that of those non-square "density matrices," referring their rows and columns to different numbers of particles, would not have been made explicit.

As a conclusion of that contraction, the $p$-FR has as a pre-image the $N$-FR which expresses the antisymmetry and normalization properties of the $N$-electron wave function. It was to be expected that the contraction of the $N$-FR would produce a condition compatible with the $p$-FR, but it is very interesting to note that the result of all these operations does not impose any new and more exacting condition. 
An important direct implication of this development is that $p$-FR may be obtained by contraction not only from the $N$-FR but also from any $m$-FR with $m>p$. Moreover, when one is interested in a $p$-RDM it is important to realize that the set of $q$-FR's relates the RDM's and HRDM's of all orders $q \leqslant p$ in a unique way. As will be seen in Sec. VII, this result is essential for establishing a testing procedure for the $N$-representability of the matrix obtained in the it-CSE.

\section{A RELIABLE RDM $N$-REPRESENTABILITY TESTING PROCEDURE WITHIN THE CSE FRAMEWORK}

The aim of this final section is to present a general outlook of the problem leading to our proposal of a succinct and reliable testing procedure for deciding upon the $\mathrm{N}$-representability of a matrix, represented in the twoelectron space, and obtained through the it-CSE.

The main characteristic of RDM's is the fact that there are tight interrelations among the elements of RDM's of different orders. The interrelations appear in families of equations such as the CSE's (Sec. III), the $G$-matrix inequalities (Sec. IV B), and the spin eigenvalue equations [33]. Moreover, the contracting mapping expressions, for holes as well as for particles, combined with the wave function antisymmetry property, generates another family of relations (Sec. VI), the $p$-FR, which must also be consistent with those other hierachical families.

As mentioned in Sec. III, the construction of rather accurate high-order RDM's from the knowledge of the 1- and 2-RDM's (when the states have a single dominant configuration) has been already achieved, and we expect that the results will still be improved in the future. Therefore, we are confident that the hierarchy relations among RDM's of different orders can be decoupled and that the it-CSE will provide reliable solutions. However, as has been mentioned, we should make sure that the 2-RDM obtained at convergence of the it-CSE is $N$ representable or, at least, closely so. Previous sections have analyzed the 2-RDM's properties which derive from the density character of its two-body operator, and from the fact that the $N$-electron state $\mathcal{L}$ is normalized and antisymmetric. This double analysis has provided a set of inequalities, some of them already very well known, which determine a set of conditions which may be classified into two categories: the conditions that the individual 2-RDM elements must satisfy and the conditions that the set of second- first-, and zero-order matrices should fulfill.

The first kind of condition must be taken into account when constructing 3- and 4-RDM's, and in fact may prove to be highly useful for this purpose. The second kind of condition which we call global, impose the positivity of the 2-RDM, 2-HRDM, 2-G, 1-RDM and 1-HRDM. Moreover, this condition imposes that these matrices are uniquely interrelated through the 2-FR 1-FR and the CM and their traces must exactly coincide with the corresponding correct values which are a function of the number of electrons $N$ and of the size of the spin-orbital basis $2 K$. The testing procedure that we therefore propose can also be decomposed into two parts

(1) The 2-RDM off-diagonal elements and the diagonal elements of the 3 and 4-RDM diagonal elements entering the CSE at the final iteration should satisfy the set of inequalities reported in Sec. IV $(31,35$, and 37, and related fourth order conditions). If that were not the case, one (or, if necessary, more than one) additional iterations should be performed. In these iterations, either the higher-order matrices or the 2-RDM off-diagonal elements should be forced to comply with these $N$-representability necessary conditions. This can be achieved by ascribing to the matrix elements the limiting value imposed by the inequalities. This control, which may be particularly useful in the case of those elements involving indices corresponding to frontier orbitals, should at least be applied in the last iteration.

(2) The following tests should also be applied when performing the final CSE iterations (a) The matrices: ${ }^{2} \mathrm{D},{ }^{1} \mathrm{D},{ }^{2} \overline{\mathrm{D}}$, and ${ }^{1} \overline{\mathrm{D}}$ must be positive and uniquely related through the CM and the 2-FR and 1-FR. (b) The traces must be very accurate. (c) The ${ }^{2} G$ matrix must be positive. (d) The pseudoidempotency inequality should be satisfied. We are aware that there are several important third and fourthorder relations which have been omitted from the testing procedure just proposed. Nevertheless, the conditions just stated are rather exacting and would not be easily met by a non $N$-representable matrix.

Note that with this testing procedure, the two essential components of the 2-RDM [Eq. (22)], the 1-RDM, and the two-body correlation matrix, or, equivalently, the ${ }^{2} G$ matrix, are controlled.

In view of the good results obtained in the numerical calculations of the high-order matrices, [31-35], and provided that each one of the controls is applied in the form of a constraint when constructing the critical elements of the high order matrices, as well as in the final test of the resulting matrices, one may safely accept the iterative solution of the 2-CSE.

Finally, let us add that this RDM theoretical investigation has led to several new and relevant relations, reported in Secs. IV and V as well as in the Appendixes.

\section{ACKNOWLEDGMENTS}

We warmly thank J. A. Coleman and R. Erdhal for their constructive comments, which are at the origin of Sec. IV. We wish to thank J. Avery, J. Fernández-Rico, J. González, D. Parry, and S. Miret for their very helpful comments and suggestions. This work was supported by the Dirección General de Investigación Científica y Técnica del Ministerio de Educación y Ciencia under Project No. PB96-0891.

\section{APPENDIX A: PRACTICAL USE OF A SET OF RDM ELEMENTS}

It is well known that when both the bra and ket of Eq. (1) [or Eq. (2)] are Slater determinants, the value of the corresponding element can only take the value 1,0 , or -1 . Since the value of one of such element can be expressed as a partially antisymmetrized sum of products of Kronecker deltas, it follows that any such antisymmetrized sum may be expressed in close form as an element of this set of RDM's. 
This property is frequently used here, which is why we deem it pertinent to give an explicit example of its application. Thus, supposing that we have the expression

$$
\left\{\delta_{i v} \delta_{j k} \delta_{t l}+\delta_{i t} \delta_{j l} \delta_{v k}-\delta_{i v} \delta_{j l} \delta_{k t}-\delta_{i t} \delta_{j k} \delta_{v l}\right\},
$$

whose value can only be 1,0 , or -1 with $v<t, k<l$; then defining $\langle\gamma| \equiv\langle t v|$ and $|k l\rangle \equiv|\lambda\rangle$ one may exactly rewrite the same expression (A1) as

$$
\left\langle\gamma\left|\mathrm{b}_{i}^{\dagger} \mathrm{b}_{j}\right| \lambda\right\rangle \equiv{ }^{1} \mathrm{D}_{i j}^{\gamma \lambda} .
$$

In this way, a combination of products of Kronecker deltas is formulated as a transition reduced density natrix where the so-called "states" are Slater determinants $\gamma$ and $\lambda$, and row and column indices label spin orbitals. Then, the rules of algebra may be used to handle these quantities.

\section{APPENDIX B: ALGEBRA OF THE GLOBAL OPERATORS B}

The use of global operators allows a very compact description of the properties concerning a group of several electron operators. Here we report a series of algebra rules which relate the global operators; they are, in our opinion, of general interest. One of them, the RDM-contracting mapping, has been repeatedly defined and used in the past but its expression in our notation is given here in order to keep the paper self-contained. Other relations given here may be known to some RDM specialists; however, to our knowledge, they have not been published or used in a similar form.

\section{Matrix contracting mappings}

(1) Contracting Mapping for RDM's. Formal properties of the contracting mapping for RDM's was given by Kummer [11]. Later on, one of us (C.V.) reported the general form of its matrix representation $[42,43,47,48]$. Here we will reproduce this latter deduction in terms of the global operators. By inserting a unit operator between the two operators of an RDM element, one has

$$
{ }^{m} \mathrm{D}_{\lambda \gamma}^{\mathcal{L}}=\left\langle\mathcal{L}\left|{ }^{m} \mathrm{~B}_{\lambda}^{\dagger m} \mathrm{~B}_{\gamma}\right| \mathcal{L}\right\rangle=\sum_{\tau} \frac{\left\langle\left.\mathcal{L}\right|^{m} \mathrm{~B}_{\lambda}^{\dagger t} \mathrm{~B}_{\tau}^{\dagger t} \mathrm{~B}_{\tau}^{m} \mathrm{~B}_{\gamma} \mid \mathcal{L}\right\rangle}{\left(\begin{array}{c}
N-m \\
t
\end{array}\right)} .
$$

Now using a procedure similar to that described in Appendix A, one may write:

$$
{ }^{m} \mathrm{D}_{\lambda \gamma}^{\mathcal{L}} \equiv \sum_{\Lambda \Omega} \frac{\left\langle\left.\Lambda\right|^{m} \mathrm{~B}_{\lambda}^{\dagger m} \mathrm{~B}_{\gamma} \mid \Omega\right\rangle}{\left(\begin{array}{c}
N-m \\
t
\end{array}\right)}\left\langle\left.\mathcal{L}\right|^{(m+t)} \mathrm{B}_{\Lambda}^{\dagger}{ }^{(m+t)} \mathrm{B}_{\Omega} \mid \mathcal{L}\right\rangle .
$$

That is,

$$
{ }^{m} \mathrm{D}_{\lambda \gamma}^{\mathcal{L}} \equiv \sum_{\Lambda \Omega} \frac{{ }^{m} \mathrm{D}_{\lambda \gamma}^{\Lambda \Omega}}{\left(\begin{array}{c}
N-m \\
t
\end{array}\right)}{ }^{(m+t)} \mathrm{D}_{\Lambda \Omega}^{\mathcal{L}}
$$

Calling $p=m+t$, one obtains the general formula of the contracting mapping for the case $N \geqslant p \geqslant m$ :

$$
{ }^{m} \mathbf{D}^{\mathcal{L}} \equiv \frac{\left(\begin{array}{c}
N \\
M
\end{array}\right)}{\left(\begin{array}{c}
p \\
m
\end{array}\right)\left(\begin{array}{c}
N \\
p
\end{array}\right)} \sum_{\Lambda \Omega}^{m} \mathbf{D}^{\Lambda \Omega p} \mathrm{D}_{\Lambda \Omega}^{\mathcal{L}} .
$$

This formula is equivalent to the integration of a $p$-RDM corresponding to an $N$-electron state over $p-m$ electron variables.

Contracting mapping for HRDM's. We will now deduce the formula of the CM for directly obtaining (without an intermediate step as above) a $p$-HRDM from the $N$-HRDM. Let us insert the $(N-p)$-hole unit operator between the $B$ and $B^{\dagger}$ in the $p$-HRDM. Thus

$$
\begin{aligned}
{ }^{p} \overline{\mathrm{D}}_{\alpha \beta}^{\mathcal{L}} & =\left\langle\mathcal{L}\left|{ }^{p} \mathrm{~B}_{\beta}{ }^{p} \mathrm{~B}_{\alpha}^{\dagger}\right| \mathcal{L}\right\rangle \\
& =\frac{1}{\left(\begin{array}{c}
2 K-(N+p) \\
N-p
\end{array}\right)} \sum_{\tau}\left\langle\left.\mathcal{L}\right|^{p} \mathrm{~B}_{\beta}{ }^{(N-p)} \mathrm{B}_{\tau}{ }^{(N-p)} \mathrm{B}_{\tau}^{\dagger p} \mathrm{~B}_{\alpha}^{\dagger} \mid \mathcal{L}\right\rangle
\end{aligned}
$$

Now, following the procedure previously described this element may be rewritten as

$$
\begin{gathered}
{ }^{p} \overline{\mathrm{D}}_{\alpha \beta}^{\mathcal{L}} \equiv \frac{1}{\left(\begin{array}{c}
2 K-(N+p) \\
N-p
\end{array}\right)} \sum_{\Lambda \Omega}^{p} \mathrm{D}_{\alpha \beta}^{\Lambda \Omega}\left\langle\left.\mathcal{L}\right|^{N} \mathrm{~B}_{\Omega}{ }^{N} \mathrm{~B}_{\Lambda}^{\dagger} \mid \mathcal{L}\right\rangle, \\
{ }^{p} \overline{\mathbf{D}}^{\mathcal{L}} \equiv \frac{1}{\left(\begin{array}{c}
2 K-(N+p) \\
N-p
\end{array}\right)} \sum_{\Lambda \Omega}{ }^{p} \mathbf{D}^{\Lambda \Omega}{ }^{N} \overline{\mathrm{D}}_{\Lambda \Omega}^{\mathcal{L}} .
\end{gathered}
$$

This relation establishes that the CM for HRDM's has the same form as RDM's except for the value of the coefficient.

\section{Normal form of global operators}

In order to obtain the normal form of a string of these operators, the most convenient approach is to generalize the procedures used when deriving relation (A2) from relation (A1).

Thus, the two main ideas at the basis of our development are as follows:

Expressing every partially antisymmetrized sum of products of Kronecker deltas as a matrix element.

Introducing sums over one or several auxiliary states, i.e., $t$ and $v$ in relation (A2), since the only terms contributing to these sums are the relevant ones.

The string of operators $\mathrm{b}_{r}^{\dagger} \mathrm{b}_{s}{ }^{p} \mathrm{~B}_{\lambda}^{\dagger}$ is a simple example, so as to show in detail the operations involved for transforming it into its normal form. Recalling that $|\lambda\rangle=\left|\lambda_{1} \lambda_{2} \ldots \lambda_{p}\right\rangle$ with $\lambda_{1}<\lambda_{2}<\cdots<\lambda_{p}$, one has

$$
\begin{aligned}
\mathrm{b}_{r}^{\dagger} \mathrm{b}_{s}^{p} \mathrm{~B}_{\lambda}^{\dagger}= & \delta_{s \lambda_{1}} \mathrm{~b}_{r}^{\dagger} \mathrm{b}_{\lambda_{2}}^{\dagger} \ldots \mathrm{b}_{\lambda_{p}}^{\dagger}-\delta_{s \lambda_{2}} \mathrm{~b}_{r}^{\dagger} \mathrm{b}_{\lambda_{1}}^{\dagger} \mathrm{b}_{\lambda_{3}}^{\dagger} \ldots \mathrm{b}_{\lambda_{p}}^{\dagger} \\
& \pm \delta_{s \lambda_{p}} \mathrm{~b}_{r}^{\dagger} \mathrm{b}_{\lambda_{1}}^{\dagger} \ldots \mathrm{b}_{\lambda_{p-1}}^{\dagger}+{ }^{p} \mathrm{~B}_{\lambda}^{\dagger} \mathrm{b}_{r}^{\dagger} \mathrm{b}_{s} .
\end{aligned}
$$

Following the previous arguments, all these terms may be collected as 


$$
\begin{aligned}
\mathrm{b}_{r}^{\dagger} \mathrm{b}_{s}{ }^{p} \mathrm{~B}_{\lambda}^{\dagger} & ={ }^{p} \mathrm{~B}_{\lambda}^{\dagger} \mathrm{b}_{r}^{\dagger} \mathrm{b}_{s}+\sum_{\pi}^{\left(\begin{array}{c}
2 K \\
p
\end{array}\right)}\left\langle\pi\left|\mathrm{b}_{r}^{\dagger} \mathrm{b}_{s}\right| \lambda\right\rangle^{p} \mathrm{~B}_{\pi}^{\dagger} \\
& ={ }^{p} \mathrm{~B}_{\lambda}^{\dagger} \mathrm{b}_{r}^{\dagger} \mathrm{b}_{s}+\sum_{\pi}^{p}{ }^{1} \mathrm{D}_{r s}^{\pi \lambda} p \mathrm{~B}_{\pi}^{\dagger}
\end{aligned}
$$

where, in spite of the $\Sigma_{\pi}$, only the terms in which $p-1$ orbitals coincide with those of $\lambda$ will contribute a nonzero value. In a similar way as in relation (B9), one obtains

$$
{ }^{p} \mathrm{~B}_{\omega} \mathrm{b}_{r}^{\dagger} \mathrm{b}_{s}=\mathrm{b}_{r}^{\dagger} \mathrm{b}_{s}{ }^{p} \mathrm{~B}_{\omega}+\sum_{\pi}^{\left(\begin{array}{c}
2 K \\
p
\end{array}\right)}{ }^{1} \mathrm{D}_{r s}^{\omega \pi}{ }^{p} \mathrm{~B}_{\pi} .
$$

By applying the same kind of arguments, the two following compact and general formulas have been obtained:

$$
\begin{aligned}
& { }^{q} \mathrm{~B}_{\mu}{ }^{p} \mathbf{B}_{\lambda}^{\dagger}=\sum_{k=0}^{q}(-1)^{(p-q) q+k}
\end{aligned}
$$

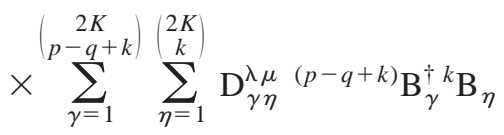

$$
\begin{aligned}
& (p>q) \\
& { }^{q} \mathbf{B}_{\mu}{ }^{p} \mathbf{B}_{\lambda}^{\dagger}=\sum_{k=0}^{p}(-1)^{(q-p) p+k} \\
& \times \sum_{\gamma=1}^{\left(\begin{array}{c}
2 K \\
k
\end{array}\right)} \sum_{\eta=1}^{\left(\begin{array}{c}
2 K \\
q-p+k
\end{array}\right)} \mathrm{D}_{\gamma \eta}^{\lambda \mu}{ }^{k} \mathrm{~B}_{\gamma}^{\dagger}{ }^{(q-p+k)} \mathrm{B}_{\eta} \\
& (p<q)
\end{aligned}
$$

Note, that when $p=q$ both relations coincide.

\section{a. External traces}

There is another important way in which the relations just seen, combined with the external traces of a string of global operators in their normal form, are used in Sec. VI. Consider

$$
\sum_{\Lambda}\left\langle\Lambda\left|{ }^{p} \mathrm{~B}_{\alpha}^{\dagger p} \mathrm{~B}_{\beta}\right| \Lambda\right\rangle
$$

where $\Lambda$ is an $N$-electron Slater determinant. It vanishes, unless $\alpha$ is equal to $\beta$.

The sum over $\Lambda$ implies that the result of this term is independent of $\alpha$. Therefore, one may sum over $\alpha$ thus obtaining $\left(\begin{array}{l}N \\ p\end{array}\right)$, and divide by the number of times that we have counted the original term, $\left(\begin{array}{c}2 K \\ p\end{array}\right)[45,46]$. Therefore,

$$
\sum_{\Lambda}{ }^{p} \mathrm{D}_{\alpha \beta}^{\Lambda \Lambda}=\delta_{\alpha \beta} \sum_{\Lambda}{ }^{p} \mathrm{D}_{\alpha \alpha}^{\Lambda \Lambda}=\delta_{\alpha \beta} \frac{\left(\begin{array}{c}
N \\
p
\end{array}\right)}{\left(\begin{array}{c}
2 k \\
p
\end{array}\right)} \sum_{\Lambda}\langle\Lambda \mid \Lambda\rangle
$$

$$
=\delta_{\alpha \beta} \frac{\left(\begin{array}{c}
N \\
p
\end{array}\right)}{\left(\begin{array}{c}
2 K \\
p
\end{array}\right)}\left(\begin{array}{c}
2 K \\
N
\end{array}\right)=\delta_{\alpha \beta}\left(\begin{array}{c}
2 K-p \\
N-p
\end{array}\right) .
$$

Let us now suppose that we have to evaluate in a closed form:

$$
\sum_{\Lambda}\left\langle\Lambda\left|{ }^{p} \mathrm{~B}_{\lambda}^{\dagger}{ }^{q} \mathrm{~B}_{\tau}^{\dagger}{ }^{r} \mathrm{~B}_{\nu}{ }^{t} \mathrm{~B}_{\mu}\right| \Lambda\right\rangle
$$

where $p+q=r+t$. This can be expressed as

$$
\begin{gathered}
\langle\nu \mu \mid \lambda \tau\rangle \sum_{\Lambda}\left\langle\left.\Lambda\right|^{(p+q)} \mathrm{B}_{\eta}^{\dagger}{ }^{(p+q)} \mathrm{B}_{\eta} \mid \Lambda\right\rangle \\
=\left(\begin{array}{c}
2 K-p-q \\
N-p-q
\end{array}\right)\left\langle\nu\left|{ }^{t} \mathrm{~B}_{\mu}{ }^{p} \mathrm{~B}_{\lambda}^{\dagger}\right| \tau\right\rangle,
\end{gathered}
$$

where $\eta$ is an arbitrary Slater-type state of $p+q$ electrons. Now we may apply Eqs. (B11) or (B12) according to whether $t<p$ or $t>p$. It should be noted that, of all the terms generated by these formulas, only those involving a global annihilator of order $u$ such that $u \leqslant q$ and a global creator of order $g$ such that $g \leqslant r$ contribute to the final value.

\section{b. A useful property of traces}

To conclude, we wish to describe another transformation which is also useful. Let us suppose that we have

$$
\sum_{\gamma \pi}\left\langle\tau\left|{ }^{q} \mathrm{~B}_{\gamma}^{\dagger r} \mathrm{~B}_{\pi}\right| \alpha\right\rangle\left\langle\nu\left|{ }^{q} \mathrm{~B}_{\gamma}^{\dagger r} \mathrm{~B}_{\pi}\right| \beta\right\rangle .
$$

That is, the operators in both matrix elements are the same. We will assume that although $r \neq q, \nu$ and $\tau$ have the same number of electrons $t$, and that $\alpha$ and $\beta$ also have the same number of electrons $w$. It follows that $t-q=w-r$. Expression (B17) is the trace of a product of nonsquare matrices but by rendering explicit the hidden subset of spin-orbitals which is common to $\tau$ and to $\alpha$ and that which is common to $\nu$ and to $\beta$ one may transform this expression into the trace of a product of two square matrices. Let us start by writing

$$
\begin{gathered}
\left\langle\tau\left|{ }^{q} \mathrm{~B}_{\gamma}^{\dagger r}{ }^{r} \mathrm{~B}_{\pi}\right| \alpha\right\rangle \equiv\left\langle x \gamma\left|{ }^{q} \mathrm{~B}_{\gamma}^{\dagger r}{ }^{r} \mathrm{~B}_{\pi}\right| \pi x\right\rangle s_{\tau ; x \gamma} s_{\alpha ; \pi x}, \\
\left\langle\nu\left|{ }^{q} \mathrm{~B}_{\gamma}^{\dagger r} \mathrm{~B}_{\pi}\right| \beta\right\rangle \equiv\left\langle y \gamma\left|{ }^{q} \mathrm{~B}_{\gamma}^{\dagger r} \mathrm{~B}_{\pi}\right| \pi y>s_{\nu ; y \gamma} s_{\beta ; \pi y},\right.
\end{gathered}
$$

where $s_{\tau ; x \gamma}$ denotes the sign following from ordering $\tau$ as $x \gamma$ and similarly for the other symbols. Hence

$$
\begin{aligned}
\sum_{\gamma \pi} & \left\langle\tau\left|{ }^{q} \mathrm{~B}_{\gamma}^{\dagger r} \mathrm{~B}_{\pi}\right| \alpha\right\rangle\left\langle\nu\left|{ }^{q} \mathrm{~B}_{\gamma}^{\dagger r} \mathrm{~B}_{\pi}\right| \beta\right\rangle \\
& \equiv \sum_{x, y}\left\langle\tau\left|{ }^{(t-q)} \mathrm{B}_{x}^{\dagger(t-q)} \mathrm{B}_{y}\right| \nu\right\rangle\left\langle\left.\alpha\right|^{(t-q)} \mathrm{B}_{x}^{\dagger(t-q)} \mathrm{B}_{y} \mid \beta\right\rangle \\
& \equiv \operatorname{tr}\left({ }^{(t-q)} \mathbf{D}^{\tau \nu}{ }^{(t-q)} \mathbf{D}^{\beta \alpha}\right) .
\end{aligned}
$$

All these intermediate results, which establish the rules for operating with global operators, render feasible the otherwise rather complicated task of contracting the $N$-FR, which is carried out in Sec. VI. 
[1] P.A.M. Dirac, Proc. Cambridge Philos. Soc. 27, 240 (1931).

[2] K. Husimi, Proc. Phys. Soc. Jpn. 22, 264 (1940).

[3] P.O. Löwdin, Phys. Rev. 97, 1474 (1955).

[4] J.E. Mayer, Phys. Rev. 100, 1579 (1955).

[5] A.J. Coleman, Rev. Mod. Phys. 35, 668 (1963).

[6] R. McWeeny, Rev. Mod. Phys. 32, 335 (1960).

[7] D. Ter Haar, Rep. Prog. Phys. 24, 304 (1961).

[8] R.H. Tredgold, Phys. Rev. 105, 1421 (1957).

[9] R.V. Ayres, Phys. Rev. 111, 1453 (1958).

[10] C. Garrod, J.K. Percus, J. Math. Phys. 5, 1756 (1964).

[11] H. Kummer, J. Math. Phys. 8, 2063 (1967).

[12] H. Kummer, I. Absar, and A.J. Coleman, J. Math. Phys. 18, 329 (1977).

[13] J.E. Harriman, Phys. Rev. A 19, 1893 (1979).

[14] J.K. Percus, Int. J. Quantum Chem. 13, 89 (1978).

[15] R.M. Erdahl, Int. J. Quantum Chem. 13, 697 (1978).

[16] A.J. Coleman and I. Absar, Int. J. Quantum Chem. 18, 1279 (1980).

[17] F. Weinhold and E.B. Wilson, J. Chem. Phys. 47, 2298 (1967).

[18] Reduced Density Matrices with Applications to Physical and Chemical Systems, Queen's Papers on Pure and Applied Mathematics No. 11 (Queen's University Press. Kingston, Ontario, 1968).

[19] M.B. Ruskai, J. Math. Phys. 11, 3218 (1970).

[20] R. Erdahl, J. Math. Phys. 13, 1608 (1972).

[21] E.R. Davidson, Reduced Density Matrices in Quantum Chemistry (Academic, New York, 1976), and references therein.

[22] W.B. McRae and E.R. Davidson, J. Math. Phys. 13, 1527 (1972).

[23] A.J. Coleman, Int. J. Quantum Chem. 13, 67 (1978).

[24] J. Simons and J.E. Harriman, Phys. Rev. A 2, 1034 (1970).

[25] B. Ruskai, Phys. Rev. A 5, 1336 (1972).

[26] A.J. Coleman, in The Force Concept in Chemistry, edited by P.M. Deb (Van Nostrand, New York, 1981).

[27] B. Weiner, Phys. Rev. A 30, 2922 (1984).

[28] J.A. Coleman, in Density Matrices and Density Functionals,
Proceedings of the A. J. Coleman Symposium, Kingston, Ontario, 1985, edited by R. Erdahl and V. Smith (Reidel, Dordrecht, 1987).

[29] P.O. Löwdin, in Density Matrices and Density Functionals (Ref. [28]), p. 1.

[30] F. Colmenero, C. Pérez del Valle, and C. Valdemoro, Phys. Rev. A 47, 971 (1993).

[31] F. Colmenero and C. Valdemoro, Int. J. Quantum Chem. 51, 369 (1994).

[32] H. Nakatsuji and K. Yasuda, Phys. Rev. Lett. 76, 1039 (1996).

[33] C. Valdemoro, L.M. Tel, E. Pérez-Romero, Adv. Quantum Chem. 28, 33 (1997).

[34] K. Yasuda and H. Nakatsuji, Phys. Rev. A 56, 2648 (1997).

[35] D. Mazziotti, Phys. Rev. A 57, 4219 (1998).

[36] D. Mazziotti, Int. J. Quantum Chem. 70, 557 (1998).

[37] C. Valdemoro, M.P. de Lara-Castells, E. Pérez-Romero, and L.M. Tel, Adv. Quantum Chem. 31, 37 (1999).

[38] C. Valdemoro, L. M. Tel, and E. Pérez-Romero, in Quantum Systems in Chemistry and Physics, Vol. 1: Basic Problems and Model Systems, edited by A. Hernandez-Laguna, J. Maruani, R. McWeeny, and S. Wilson (Kluwer, Dordrecht, 2000).

[39] C. Valdemoro, in Topics in Current Chemistry, "Correlation and Localization.', edited by P. Surjan (Springer-Verlag, Berlin, 1999).

[40] H. Nakatsuji, Phys. Rev. A 14, 41 (1976).

[41] L. Cohen and C. Frishberg, Phys. Rev. A 13, 927 (1976).

[42] C. Valdemoro, in Density Matrices and Density Functionals (Ref. [27]) p. 275.

[43] C. Valdemoro, Phys. Rev. A 31, 2114 (1985).

[44] K. Yasuda, Phys. Rev. A 59, 4133 (1999).

[45] D. Mazziotti, Phys. Rev. A 60, 3618 (1999).

[46] C. Valdemoro, Phys. Rev. A 45, 4462 (1992).

[47] C. Valdemoro, An. R. Soc. Esp. Fis. Quim. 79, 106 (1983).

[48] C. Valdemoro, in Strategies and Applications in Quantum Chemistry, edited by Y. Ellinger and M. Defranceschi (Klüwer, Dordrecht, 1996) p. 55. 